\title{
Semi-Lagrangian schemes for the Vlasov equation on an unstructured mesh of phase space
}

\author{
N. Besse ${ }^{a, b}$ \\ ${ }^{a}$ Commissariat à l'Energie Atomique, \\ DAM-Ile de France, BP No 12, \\ 91680 Bruyères-le-Châtel \\ France. \\ ${ }^{\mathrm{b}}$ Institut de Recherche Mathematique Avancée, \\ Université Louis Pasteur - CNRS, \\ 7 rue René Descartes, \\ 67084 Strasbourg Cedex, France
}

\begin{abstract}
A new scheme for solving the Vlasov equation using an unstructured mesh (triangulation) for the phase space is proposed. The algorithm is based on the semiLagrangian method which exploits the fact that the distribution function is constant along the characteristic curves. We use different local interpolation operators to reconstruct the distribution function $f$, some of which need the knowledge of the gradient of $f$. We can use limiter coefficients to maintain the positivity and the $L^{\infty}$ bound of $f$ and optimize these coefficients to ensure the conservation of the $L^{1}$ norm that is to say the mass by solving a linear programming problem. Several numerical results are presented in two and three (axisymmetric case) dimensional phase space. The local interpolation technique is well suited for parallel computation.
\end{abstract}

Key words: Vlasov-Poisson system, semi-Lagrangian methods, conservative laws, plasma physics, particle beams, time splitting.

Email address: (a) nicolas.besse@cea.fr, (b) besse@math.u-strasbg.fr (N. Besse). 


\section{Introduction.}

The Vlasov equation describes the evolution of a system of particles under the effects of self-consistent and applied electromagnetic fields. The unknown $f(t, x, v)$, where $t$ stand for time, $x$ for position and $v$ for velocity, represents the distribution function of particles (ions, electrons,...) in phase space. The Vlasov equation is used to study collisionless plasma and propagation of charged particle beams.

The numerical resolution of the Vlasov equation is most of the time performed by Lagrangian methods like particle in cell methods (PIC) which consist of approximating the plasma by a finite number of macro-particles. The trajectories of these particles are computed from characteristic curves given by the Vlasov equation, whereas self-consistent fields are computed by gathering the charge and current densities of the the particles on a mesh of the physical space (see Birdsall and Langdon for more details [1]). Although this method allows us to obtain satisfying results with a small number of particles, it is well known that the numerical noise inherent to the particle method becomes too significant to allow a precise description of the tail of the distribution function which plays an important role in charged particle beams. This numerical noise decreases in $1 / \sqrt{N}$ when $N$, the number of particles increases. To remedy this problem, Eulerian methods which consist in discretizing the Vlasov equation on a mesh of phase space have been proposed. For example a finite element method has been proposed in [22]. Although this method takes into account complicated boundaries, it requires to solve a system which makes it hard to use it in high dimension as global resolution is too long. Another method called Fourier-Fourier transform, based on the Fourier transform of the distribution function in phase space, works for periodic boundaries condition, but for non periodic problems Gibbs oscillations become a source of spurious oscillations which propagate into the distribution function (see [11]). Other methods like the flux corrected transport (FCT) [2], and the flux balance method (FBM) [15], [8] is based on the computation of the average of the Vlasov equation on each cell of the grid by a conservative methods like finite volume method. The techniques used to reconstruct the distribution function do not preserve the positivity which is an inconvenient for lengthy simulations. The positive flux conservative method (PFC) [9] proposes a reconstruction which preserves positivity and mass by introducing slope limiters. Nevertheless these methods are low order and dissipative. Other kind of Eulerian method are the semiLagrangian method (see Sonnendrücker et al [20]) consisting in computing directly the distribution function on a Cartesian grid of the phase space. This computation is done by integrating the characteristics curves backward at each time step and interpolating the value at the feet of the characteristics by a cubic spline method. Nakamura and Yabe proposed the cubic interpolated propagation (CIP) method based on the approximation of the gradients of the distribution function in order to use the one dimensional Hermite interpola- 
tion.

In this paper we propose a new method based on the semi-Lagrangian principle for solving the Vlasov equation on an unstructured mesh in phase space. This method works with different kinds of high order local interpolation operators requiring the knowledge of the gradients which are obtained by advecting them. Moreover we use ideas developed in numerical weather prediction codes by Priestley [17] and Staniforth [19] to get a positive and conservative method by introducing a local linear combination of low order solution and high order solution.

This paper is organized as follows. In the first part we recall the Vlasov equation and some properties of the solution. Then we present the numerical method based on semi-Lagrangian ideas. In the last section we present numerical results in two and three dimensional phase space in the domain of collisionless plasma and charged particle beams.

\section{The Vlasov equation}

The evolution of the distribution function of particles $f(t, x, v)$ in phase space $(x, v) \in \mathbb{R}^{d} \times \mathbb{R}^{d}, d=1, \ldots, 3$, is given by the Vlasov equation

$$
\frac{\partial f}{\partial t}+v \cdot \nabla_{x} f+F(t, x, v) \cdot \nabla_{v} f=0
$$

where the force field $F(t, x, v)$ is coupled with the distribution function $f$ giving a non linear system. We recall two well known models, Vlasov-Poisson (VP) and Vlasov-Maxwell (VM) which describes the evolution of charged particles under the effects of self-consistent electromagnetic fields.

The coupling between $f$ and the force field is done by the source terms $\rho$, the charge density and $j$ the current density which are given by

$$
\rho(t, x)=q \int_{\mathbf{R}^{d}} f(t, x, v) d v, \quad j(t, x)=q \int_{\mathbf{R}^{d}} v f(t, x, v) d v .
$$

For the (VP) system the force field is given by

$$
F(t, x, v)=\frac{q}{m} E(t, x), \quad E(t, x)=-\nabla_{x} \phi(t, x), \quad-\varepsilon_{0} \Delta \phi(t, x)=\rho(t, x)
$$

where $q$ and $m$ is respectively the charge and the mass of one particle. For the (VM) system the field force is given by

$$
F(t, x, v)=\frac{q}{m}(E(t, x)+v \wedge B(t, x))
$$


where $E$ and $B$ solve the Maxwell equations

$$
\left\{\begin{array}{l}
\frac{\partial E}{\partial t}-c^{2} \operatorname{curl} B=-\frac{1}{\varepsilon_{0}} j, \\
\frac{\partial B}{\partial t}+\operatorname{curl} E=0, \\
\operatorname{div}_{x} E=\frac{1}{\varepsilon_{0}} \rho, \quad \operatorname{div}_{x} B=0 .
\end{array}\right.
$$

with the compatibity condition

$$
\frac{\partial \rho}{\partial t}+\operatorname{div}_{x} j=0
$$

which is verified by integrating the Vlasov equation with respect to the variable $v$.

Now we recall the classical a priori estimates for the (VP) and (VM) system. If $f_{0}(x, v)$ is positive then $f(t, x, v)$ remains positive for all $t \geq 0$. By observing that $\operatorname{div}_{v} F(t, x, v)=0$, for all function $\beta \in C^{1}\left(\mathbb{R}^{+}, \mathbb{R}^{+}\right)$,

$$
\int_{\mathbf{R}^{d} \times \mathbb{R}^{d}} \beta(f(t, x, v)) d x d v
$$

is constant for all $t \geq 0$. Especially, all $L^{p}$ norms, $1 \leq p \leq \infty$, are preserved. Moreover if we take $\beta(r)=r \ln r$, we get the conservation of the kinetic entropy defined by

$$
H(t)=\int_{\mathbf{R}^{d} \times \mathbb{R}^{d}} f(t, x, v) \ln f(t, x, v) d x d v, \quad \forall t>0 .
$$

Next, multiplying the Vlasov equation by $|v|^{2}$, and integrating by parts we find the conservation of energy which is given by

$$
\frac{m}{2} \int_{\mathbf{R}^{d} \times \mathbb{R}^{d}} f(t, x, v)|v|^{2} d x d v+\frac{\varepsilon_{0}}{2} \int_{\mathbf{R}^{d}}|E(t, x)|^{2} d x, \quad \forall t>0,
$$

for the (VP) system and

$$
\frac{m}{2} \int_{\mathbb{R}^{d} \times \mathbf{R}^{d}} f(t, x, v)|v|^{2} d x d v+\varepsilon_{0} \int_{\mathbf{R}^{d}} \frac{|E(t, x)|^{2}+c^{2}|B(t, x)|^{2}}{2} d x, \quad \forall t>0, \quad \varepsilon_{0} \mu_{0} c^{2}=1 .
$$

for the (VM) system.

If $a(t, x, v)=(v, F(t, x, v))^{T}$ is sufficiently regular, then we can define unique characteristic curves $(X(s, x, v ; t), V(s, x, v ; t))$ of the first order differential operator,

$$
\frac{\partial}{\partial t}+a \cdot \nabla
$$


which solve the following system of ordinary differential equations

$$
\left\{\begin{array}{l}
\frac{d X}{d t}(s, x, v ; t)=V(s, x, v ; t), \\
\frac{d V}{d t}(s, x, v ; t)=F(t, X(s, x, v ; t), V(s, x, v ; t)), \\
X(s, x, v ; s)=x, V(s, x, v ; s)=v,
\end{array}\right.
$$

where $(X(s, x, v ; t), V(s, x, v ; t))$ denotes the position in phase space at the time $t$, of a particle which was at $(x, v)$ at instant $s$.

Since $\operatorname{div}_{(x, v)} a=0$, we can rewrite the Vlasov equation in the conservative form

$$
\frac{\partial f}{\partial t}+\operatorname{div}_{(x, v)}(a f)=0, \quad \forall(t, x, v) \in \mathbb{R}^{+} \times \mathbb{R}^{d} \times \mathbb{R}^{d},
$$

with the initial condition

$$
f(0, x, v)=f_{0}(x, v) .
$$

In [3] it is proved that the jacobian $J(s, x, v ; t)=\operatorname{det}\left(\partial_{(x, v)}(X(s, x, v ; t), V(s, x, v ; t))\right.$ remains positive, bounded and verify the equation

$$
\frac{\partial J}{\partial t}=J\left(\operatorname{div}_{(x, v)} a\right)(t, X(s, x, v ; t), X(s, x, v ; t)) .
$$

Then is also proved that the solution of (3) is given by

$$
f(t, x, v)=f(s, X(t, x, v ; s), V(t, x, v ; s)) J(t, x, v ; s) .
$$

In the case of Vlasov equation, as $\operatorname{div}_{(x, v)} a=0$, we get $J=1$, and the flow $(X(s, ., . ; t), V(s, ., . ; t))$ preserves the measure of the volume of the phase space so that the equation (5) becomes

$$
f(t, x, v)=f(s, X(t, x, v ; s), V(t, x, v ; s)) .
$$

This last equation means that the distribution function $f$ is constant along the characteristic curves.

Finally we mention that the structure of the Vlasov-Poisson system in six dimension is very close to the structure of the incompressible Euler equations in two dimensions written in vorticity formulation by introducing the stream function

$$
\psi(t, x)=\frac{m|v|^{2}}{2}+\frac{\phi}{2}(t, x) .
$$

which is the Halmitonian of the system. 


\section{The numerical scheme}

In this section we present our numerical scheme, which is not restricted by CFL (Courant-Friedrichs-Levy) condition as it is usually the case for the most classical Eulerian algorithms such as finite difference or finite volume schemes. The Vlasov-Poisson and the Vlasov-Maxwell models are mixing systems whose evolution can become tremendously complex. Indeed the function remains constant along the trajectories but the characteristic curves becomes more and more intricate. Then different regions of phase space where $f$ has different values come close together and steep gradients appear. The filamentation development is a problem as we loose information when the size of the filaments becomes smaller than the size of the cells. An unstructured mesh can present some advantages. First as the mesh is unstructured there is no privileged direction which could introduce a slant in physical results. If the physical system provides isolated structures with complex boundaries in phase space, an unstructured mesh allows to compute the solution only on the useful part of the phase space. Moreover unstructured meshes like triangulation is very suited for local mesh refinement and adaptative mesh refinement. Adaptative mesh refinement seems to be a good idea to follow the development of thin or microscopic structures and consequently to have a good description of the organized or coherent structures which emerge at a macroscopic level. This kind of method seems to allow to increase the ratio precision over computation time since we use few points where the solution is smooth and a greater number of points where the solution develops steep gradients.

The discretization in time is based on Strang splitting schemes which consists in solving successively a half advection in physical space, an advection in velocity space and another half advection in physic space. We advect not only the distribution function $f$ but also the gradients of $f$ because we need them to reconstruct $f$ everywhere. The equations to update the gradients is obtained by derivating the solution of the transport equation. In other words the gradients are solution of a transport problem too. Each advection is easy to perform since the characteristic curves can be solved exactly. The error of time discretization comes from the way we carry out the splitting (see [12]). First we describe the algorithm which gives the semi-discretization in time of the numerical scheme and allows to go from time step $t^{n}$ to $t^{n+1}$. 
1. Perform a half time step advection in physical space:

$$
\begin{aligned}
f^{\star}(x, v) & =f\left(t^{n}, x-v \Delta t / 2, v\right), \\
\nabla_{x} f^{\star}(x, v) & =\nabla_{x}\left(f\left(t^{n}, x-v \Delta t / 2, v\right)\right), \\
& =\nabla_{x} f\left(t^{n}, x-v \Delta t / 2, v\right), \\
\nabla_{v} f^{\star}(x, v) & =\nabla_{v}\left(f\left(t^{n}, x-v \Delta t / 2, v\right)\right), \\
& =-\frac{\Delta t}{2} \nabla_{x} f\left(t^{n}, x-v \Delta t / 2, v\right)+\nabla_{v} f\left(t^{n}, x-v \Delta t / 2, v\right)
\end{aligned}
$$

2. Compute the electric field $E^{\star}(x)$ by substituting $f^{\star}$ in the Poisson equation that is to say solve the following system:

$$
\begin{cases}E^{\star}(x) & =-\nabla_{x} \phi^{\star}(x) \\ -\Delta \phi^{\star}(x) & =\rho^{\star}(x) \\ \rho^{\star}(x) & =\int_{\mathbf{R}^{d}} f^{\star}(x, v) d v,\end{cases}
$$

3. Perform a step time advection in velocity space:

$$
\begin{aligned}
f^{\star \star}(x, v) & =f^{\star}\left(x, v-E^{\star}(x) \Delta t\right), \\
\nabla_{x} f^{\star \star}(x, v) & =\nabla_{x}\left(f^{\star}\left(x, v-E^{\star}(x) \Delta t\right)\right), \\
& =\nabla_{x} f^{\star}\left(x, v-E^{\star}(x) \Delta t\right)-\Delta t \nabla E^{\star T}(x) \nabla_{v} f^{\star}\left(x, v-E^{\star}(x) \Delta t\right), \\
\nabla_{v} f^{\star \star}(x, v) & =\nabla_{v}\left(f^{\star}\left(x, v-E^{\star}(x) \Delta t\right)\right), \\
& =\nabla_{v} f^{\star}\left(x, v-E^{\star}(x) \Delta t\right)
\end{aligned}
$$

4. Perform a second half time step advection in physical space:

$$
\begin{aligned}
f\left(t^{n+1}, x, v\right) & =f^{\star \star}(x-v \Delta t / 2, v), \\
\nabla_{x} f\left(t^{n+1}, x, v\right) & =\nabla_{x}\left(f^{\star \star}(x-v \Delta t / 2, v)\right), \\
& =\nabla_{x} f^{\star \star}(x-v \Delta t / 2, v), \\
\nabla_{v} f\left(t^{n+1}, x, v\right) & =\nabla_{v}\left(f^{\star \star}(x-v \Delta t / 2, v)\right), \\
& =-\frac{\Delta t}{2} \nabla_{x} f^{\star \star}(x-v \Delta t / 2, v)+\nabla_{v} f^{\star \star}(x-v \Delta t / 2, v)
\end{aligned}
$$


At each advection step, once we have followed the characteristic curves backward we have to evaluate the function distribution $f$ and its gradients at the end points of the characteristic curves which are inevitably not the nodes of the mesh where we know $f$. Then we have to reconstruct $f$ by an interpolation technique. Next, we present different local interpolation operator which are used to reconstruct $f$. Some of them need only the knowledge of $f$ at the mesh nodes like Lagrange interpolation but for other we need to know not only $f$ but also $\nabla f$ at the mesh points.

Note that in time step 3 of the algorithm we have to know the gradient of the electric field which is a matrix $2 \times 2$ in two dimensions and a matrix $3 \times 3$ in three dimensions. In the numerical cases we present further, we do not need to compute directly the gradient of the electric field or the gradient of the force applied to the particles because we can deduce it from other physical quantities. Nevertheless, in the general case we have to compute the gradient of the force applied to the particles. In context of the coupling with the Poisson equation, as the force applied to the particles is the electric force, and since the Poisson problem is linear we obtain the gradient of the electric field by derivating the Poisson system. If $x_{i}, i=1, . ., 3$ stands for the coordinate in the $\mathrm{i}-t h$ dimension, then the derivative of the electric field $E^{\star}(x)$ with respect to the variable $x_{i}$ satisfies the following system

$$
\begin{cases}\partial_{x_{i}} E^{\star}(x) & =-\nabla_{x}\left(\partial_{x_{i}} \phi^{\star}(x)\right) \\ -\Delta\left(\partial_{x_{i}} \phi^{\star}(x)\right) & =\partial_{x_{i}} \rho^{\star}(x) \\ \partial_{x_{i}} \rho^{\star}(x) & =\int_{\mathbb{R}^{d}} \partial_{x_{i}} f^{\star}(x, v) d v\end{cases}
$$

which for example can be solved by finite element methods.

Let $\Omega$ be the compact set of $\mathbb{R}_{x} \times \mathbb{R}_{v}$ where we want to compute the solution, and $\mathcal{T}_{h}$ be a triangulation of $\Omega$. An element $T$, of the triangulation is a 2 -simplex of $\mathbb{R}^{2}$ defined by its three vertex $\left\{a_{i}\right\}_{i=1, . ., 3}$. Every point $P$ of $\mathbb{R}^{2}$ is characterized by its Cartesian coordinates $(x, v)$ and its barycentric coordinates $\lambda_{j}=\lambda_{j}(x, v), 1 \leq j \leq 3$, which are linked by the equation

$$
\forall(x, v) \in \mathbb{R}^{2} \quad(x, v)=\sum_{j=1}^{3} \lambda_{j}(x, v) a_{j} .
$$

We present first Lagrange interpolation, and secondly local interpolation techniques which involve $f$ and $\nabla f$. Most of these interpolation techniques come from the finite element literature [6]. 


\subsection{Lagrange interpolation}

In order to define the Lagrange interpolation of order $k$ on a triangle $T$, we need to define the principal lattice of order $k$ which is the set of the points of $\mathbb{R}^{2}$ determined by

$$
L_{k}(T)=\left\{(x, v) \in \mathbb{R}^{2} ; \lambda_{j}(x, v) \in\left\{0, \frac{1}{k}, \ldots, \frac{k-1}{k}, 1\right\}, 1 \leq j \leq 3\right\}
$$

where the barycentric coordinates are defined with respect to the vertex $\left\{a_{i}\right\}_{i=1, . ., 3}$. Every point of the principal lattice $L_{k}(T)$ can be written as

$$
a_{\mu}=\frac{1}{k} \sum_{j=1}^{3} \mu_{j} a_{j}, \mu=\left(\mu_{1}, \mu_{2}, \mu_{3}\right)
$$

where the coefficients $\mu_{j}$ are integer which verify the relations

$$
\mu_{j} \geq 0, \quad \sum_{j=1}^{3} \mu_{j}=k .
$$

Now we associate $L_{k}(T)$ with the set of degrees of freedom $\Sigma_{k}(T)$ defined as follows

$$
\Sigma_{k}(T)=\left\{f\left(a_{\mu}\right), a_{\mu} \in L_{k}(T)\right\}
$$

We associate $L_{k}(T)$ of cardinal $\frac{(2+k) !}{2 ! k !}$ with the space of Lagrange polynomial of order $k, P_{k}(T)$ whose dimension is $\frac{(2+k) !}{2 ! k !} . P_{k}(T)$ is defined by its basis functions $\varphi_{\mu}$ as it follows. We associate every vertex $a_{\mu}$ of $L_{k}(T)$ with the basis function $\varphi_{\mu}$ defined by

$$
\varphi_{\mu}(x, v)=\left(\prod_{j=1}^{3}\left(\mu_{j} !\right)\right)^{-1} \prod_{\substack{j=1 \\ \mu_{j} \geq 1}}^{3} \prod_{i=0}^{\mu_{j}-1}\left(k \lambda_{j}(x, v)-i\right) .
$$

The triple $\left(T, P_{k}(T), \Sigma_{k}(T)\right)$ is called a Lagrange finite element. Then we introduce the local interpolation operator $\Pi_{T}$ defined by

$$
\left(\Pi_{T} f\right)(x, v)=\sum_{\mu=1}^{N_{k}} f\left(a_{\mu}\right) \varphi_{\mu}(x, v) \quad \forall f \in C^{0}(\bar{T})
$$

with $N_{k}=\frac{(2+k) !}{2 ! k !}$ and the $\varphi_{\mu}$ defined by (6). Now we introduce the space $X_{h}$, which will be the space of the discretization of the solution.

$$
X_{h}=\left\{f \in C^{0}(\bar{\Omega}) \cap H^{1}(\Omega) ; f_{\left.\right|_{j}} \in P_{k}\left(T_{j}\right) \forall T_{j} \in \mathcal{T}_{h}\right\} .
$$

The space $X_{h}$ is characterized by the basis functions $\left\{\psi_{k}\right\}$, which verify the property $\psi_{k}\left(x_{i}, v_{i}\right)=\delta_{i, k}$, where $\left(x_{i}, v_{i}\right)$ is the $i t h$ point of the triangulation 
$\mathcal{T}_{h}$. Then, every function $f \in X_{h}$ is given by

$$
f(x, v)=\sum_{k} f\left(x_{k}, v_{k}\right) \psi_{k}(x, v) .
$$

Finally we define the global interpolation operator $\Pi_{h}$ by

$$
\left(\Pi_{h} f\right)(x, v)=\sum_{k=1}^{N} f_{k} \psi_{k}(x, v) \quad \forall f \in C^{0}(\bar{\Omega})
$$

where $f_{k}=f\left(x_{k}, v_{k}\right)$ and $N$ is the number of all degrees of freedom.

The relationship of paramount importance between the global interpolation operator $\Pi_{h}$ and the local interpolation operator $\Pi_{T}$ is given by

$$
\left(\Pi_{h} v\right)_{\left.\right|_{T}}=\Pi_{T}\left(v_{\left.\right|_{T}}\right), \quad \forall T \in \mathcal{T}_{h}, \quad v \in C^{0}(\bar{\Omega})
$$

where $v_{\left.\right|_{T}}$ denotes the restriction of $v$ onto $T$.

In fact Lagrange interpolation of degree equal or greater than three does not provide a good reconstruction for the distribution function since the Lagrange interpolation operator is not stable in the sense that there is no constant $C$ such that

$$
\left\|\Pi_{h} g\right\|_{L^{p}} \leq(1+C h)\|g\|_{L^{p}}, \quad p \in[1, \infty],
$$

where $h$ is the maximum diameter of the triangles. As the stability of the method comes from the stability of the interpolation operator, then the method is unstable. On the other hand for degree one or two the Lagrange interpolation is two dissipative. Therefore we now consider in Hermite type interpolation.

\subsection{Hermite interpolation}

In order to use local interpolation operators of finite the element method we have to define the triple $\left(T, P_{T}, \Sigma_{T}\right)$. Depending on whether the solution belong to $C^{0}(\bar{\Omega})$ or $C^{1}(\bar{\Omega})$, the space of discretization of the solution will be respectively

$$
X_{h}=\left\{f \in C^{0}(\bar{\Omega}) \cap H^{1}(\Omega) ; f_{\left.\right|_{T_{j}}} \in P_{T}\left(T_{j}\right) \forall T_{j} \in \mathcal{T}_{h}\right\}
$$

or

$$
Y_{h}=\left\{f \in C^{1}(\bar{\Omega}) \cap H^{2}(\Omega) ; f_{\left.\right|_{j}} \in P_{T}\left(T_{j}\right) \forall T_{j} \in \mathcal{T}_{h}\right\} .
$$

Then we present different local interpolation operators which require the knowledge of $f$ and $\nabla f$. In fact we just have to define $\Sigma_{T}$ and $\Pi_{T}$. Finally we denote respectively by $P_{2}$ and $P_{3}$ the sets of polynomials of degree two and three. 


\subsubsection{The $C^{0}$ reduced Hermite element ( $\mathrm{HCO}$ ).}

The set of the degrees of freedom is given by

$$
\Sigma_{T}=\left\{f\left(a_{i}\right): 1 \leq i \leq 3 ; \partial_{x} f\left(a_{i}\right), \partial_{v} f\left(a_{i}\right): 1 \leq i \leq 3\right\},
$$

and the local interpolation operator $\Pi_{T}$ is determined for all $f$ in $C^{0}(\bar{T})$ by

$$
\begin{aligned}
& \Pi_{T} f=\sum_{i=1}^{3} f\left(a_{i}\right)\left(-2 \lambda_{i}^{3}+3 \lambda_{i}^{2}+2 \lambda_{i} \lambda_{j} \lambda_{k}\right)+ \\
& \quad \frac{\lambda_{i} \lambda_{j}}{2}\left(\lambda_{i}-\lambda_{j}+1\right) \nabla f\left(a_{i}\right) \cdot\left(a_{j}-a_{i}\right)+\frac{\lambda_{i} \lambda_{k}}{2}\left(\lambda_{i}-\lambda_{k}+1\right) \nabla f\left(a_{i}\right) \cdot\left(a_{k}-a_{i}\right)
\end{aligned}
$$

where $\left\{\lambda_{i}\right\}_{i=1, . ., 3}$ are the barycentric coordinates, $j=i \bmod 3+1$, and $k=$ $j \bmod 3+1$. Note that $P_{2} \subset P_{T} \subset P_{3}$

\subsubsection{The $C^{0}$ cubic Nielson element (NCO).}

The set of the degrees of freedom is given by

$$
\Sigma_{T}=\left\{f\left(a_{i}\right): 1 \leq i \leq 3 ; \partial_{x} f\left(a_{i}\right), \partial_{v} f\left(a_{i}\right): 1 \leq i \leq 3\right\},
$$

and the local interpolation operator $\Pi_{T}$ is determined for all $f$ in $C^{0}(\bar{T})$ by

$$
\begin{aligned}
& \Pi_{T} f=\sum_{i=1}^{3} f\left(a_{i}\right)\left(-2 \lambda_{i}^{3}+3 \lambda_{i}^{2}+2 \lambda_{i} \lambda_{j} \lambda_{k}\right)+ \\
& \quad \lambda_{i} \lambda_{j}\left(\lambda_{i}+\frac{1}{2} \lambda_{k}\right) \nabla f\left(a_{i}\right) \cdot\left(a_{j}-a_{i}\right)+\lambda_{i} \lambda_{k}\left(\lambda_{i}+\frac{1}{2} \lambda_{j}\right) \nabla f\left(a_{i}\right) \cdot\left(a_{k}-a_{i}\right),
\end{aligned}
$$

where, $j=i \bmod 3+1, k=j \bmod 3+1$, and $P_{2} \subset P_{T} \subset P_{3}$.

\subsubsection{The $C^{1}$ cubic Nielson element (NC1).}

The set of the degrees of freedom is given by

$$
\Sigma_{T}=\left\{f\left(a_{i}\right): 1 \leq i \leq 3 ; \partial_{x} f\left(a_{i}\right), \partial_{v} f\left(a_{i}\right): 1 \leq i \leq 3\right\},
$$

and the local interpolation operator $\Pi_{T}$ is determined for all $f$ in $C^{1}(\bar{T})$ by

$$
\begin{aligned}
\Pi_{T} f= & \sum_{i=1}^{3} f\left(a_{i}\right)\left(\lambda_{i}^{2}\left(3-2 \lambda_{i}\right)+6 w \lambda_{i}\left(\lambda_{k} \alpha_{i j}+\lambda_{j} \alpha_{i k}\right)\right)+ \\
& \nabla f\left(a_{i}\right) \cdot\left(a_{j}-a_{i}\right)\left[\lambda_{i}^{2} \lambda_{j}+w \lambda_{i}\left(3 \lambda_{j} \alpha_{i k}+\lambda_{k}-\lambda_{j}\right)\right]+ \\
& \nabla f\left(a_{i}\right) \cdot\left(a_{k}-a_{i}\right)\left[\lambda_{i}^{2} \lambda_{k}+w \lambda_{i}\left(3 \lambda_{k} \alpha_{i j}+\lambda_{j}-\lambda_{k}\right)\right],
\end{aligned}
$$


where

$$
\begin{gathered}
w=\frac{\lambda_{1} \lambda_{2} \lambda_{3}}{\lambda_{1} \lambda_{2}+\lambda_{2} \lambda_{3}+\lambda_{1} \lambda_{3}}, \\
\alpha_{i j}=\frac{\left\|e_{i}\right\|^{2}+\left\|e_{j}\right\|^{2}-\left\|e_{k}\right\|^{2}}{2\left\|e_{j}\right\|^{2}},
\end{gathered}
$$

and $\left\|e_{i}\right\|$ denote the length of edge $e_{i}$ opposite to the vertex $a_{i}$. We have the relationship $P_{2} \subset P_{T} \subset P_{3}$.

\subsubsection{The $C^{1}$ cubic Hsieh-Clough-Tocher element(HCT-C)}

If $a_{i}$ is a vertex of a triangle $T$, then we denote respectively by $l_{i}$, and $m_{i}$ the length and the middle of the edge of $T$ opposite to the vertex $a_{i}$. We denote by $h_{i}$ the intersection point of the edge opposite to the vertex $a_{i}$ and the perpendicular to this edge which goes through $a_{i}$. Then we introduce $n_{i}=\left|h_{i}-a_{i}\right|$ and $\nu_{i}$ the unit exterior normal of the edge opposite to $a_{i}$. Let $a$ be the barycentre of $T$, then $K_{i}$ denotes the subtriangle of $T$ built with the vertex $a, a_{j}$, and $a_{k}$ where $1 \leq i \leq 3, j=i \bmod 3+1$ and $k=j \bmod 3+1$. Finally we introduce the so-called "eccentricity parameters" $e_{i}$ defined by

$$
e_{i}=\frac{l_{k}^{2}-l_{j}^{2}}{l_{i}^{2}}
$$

The set of the degrees of freedom is given by

$$
\Sigma_{T}=\left\{f\left(a_{i}\right): 1 \leq i \leq 3 ; \partial_{x} f\left(a_{i}\right), \partial_{v} f\left(a_{i}\right), \partial_{\nu_{i}} f\left(m_{i}\right): 1 \leq i \leq 3\right\},
$$

where $\partial_{\nu_{i}}$ denote the normal derivative. We can replace equivalently $\Sigma_{T}$ par $\Sigma_{T}^{\prime}$ where

$$
\Sigma_{T}^{\prime}=\left\{f\left(a_{i}\right): 1 \leq i \leq 3 ; \partial_{x} f\left(a_{i}\right), \partial_{v} f\left(a_{i}\right), \partial_{x} f\left(m_{i}\right), \partial_{v} f\left(m_{i}\right): 1 \leq i \leq 3\right\} .
$$

The local interpolation operator $\Pi_{T}$ is determined for all $f$ in $C^{1}(\bar{T})$ by

$$
\Pi_{T} f_{\left.\right|_{K_{l}}}=\sum_{i=l}^{(l+1) \bmod 3+1} f\left(a_{i}\right) \Psi_{l, i}^{0}+\left(\nabla f\left(a_{i}\right) \cdot \overrightarrow{a_{i} a_{k}}\right) \Psi_{l, i, k}^{1}+\left(\nabla f\left(a_{i}\right) \cdot \overrightarrow{a_{i} a_{j}}\right) \Psi_{l, i, j}^{1}+\left(\nabla f\left(m_{i}\right) \cdot \overrightarrow{h_{i} a_{i}}\right) \Psi_{\perp, l, i}^{1}
$$

or by

$\Pi_{T} f_{\left.\right|_{K_{l}}}=\sum_{i=l}^{(l+1) \bmod 3+1} f\left(a_{i}\right) \Psi_{l, i}^{0}+\left(\nabla f\left(a_{i}\right) \cdot \overrightarrow{a_{i} a_{k}}\right) \Psi_{l, i, k}^{1}+\left(\nabla f\left(a_{i}\right) \cdot \overrightarrow{a_{i} a_{j}}\right) \Psi_{l, i, j}^{1}-\left(n_{i} \frac{\partial f}{\partial \nu_{i}}\left(m_{i}\right)\right) \Psi_{\perp, l, i}^{1}$.

The basis functions $\left\{\Psi_{l}\right\}$ are defined by

$$
\Xi_{l}=\Sigma_{l} \Lambda_{l}
$$


with $i=l, j=i \bmod 3+1$ and $k=j \bmod 3+1$

$$
\begin{gathered}
\Xi_{l}=\left(\Psi_{l, i}^{0}, \Psi_{l, j}^{0}, \Psi_{l, k}^{0}, \Psi_{l, i, k}^{1}, \Psi_{l, i, j}^{1}, \Psi_{l, j, i}^{1}, \Psi_{l, i, k}^{1} \Psi_{l, k, j}^{1}, \Psi_{l, k, i}^{1}, \Psi_{\perp, l, i}^{1}, \Psi_{\perp, l, j}^{1}, \Psi_{\perp, l, k}^{1}\right)^{T} \\
\Lambda_{l}=\left(\lambda_{i}^{3}, \lambda_{j}^{3}, \lambda_{k}^{3}, \lambda_{i}^{2} \lambda_{k}, \lambda_{i}^{2} \lambda_{j}, \lambda_{j}^{2} \lambda_{i}, \lambda_{j}^{2} \lambda_{k}, \lambda_{k}^{2} \lambda_{j}, \lambda_{k}^{2} \lambda_{i}, \lambda_{i} \lambda_{j} \lambda_{k}\right)^{T}
\end{gathered}
$$

and

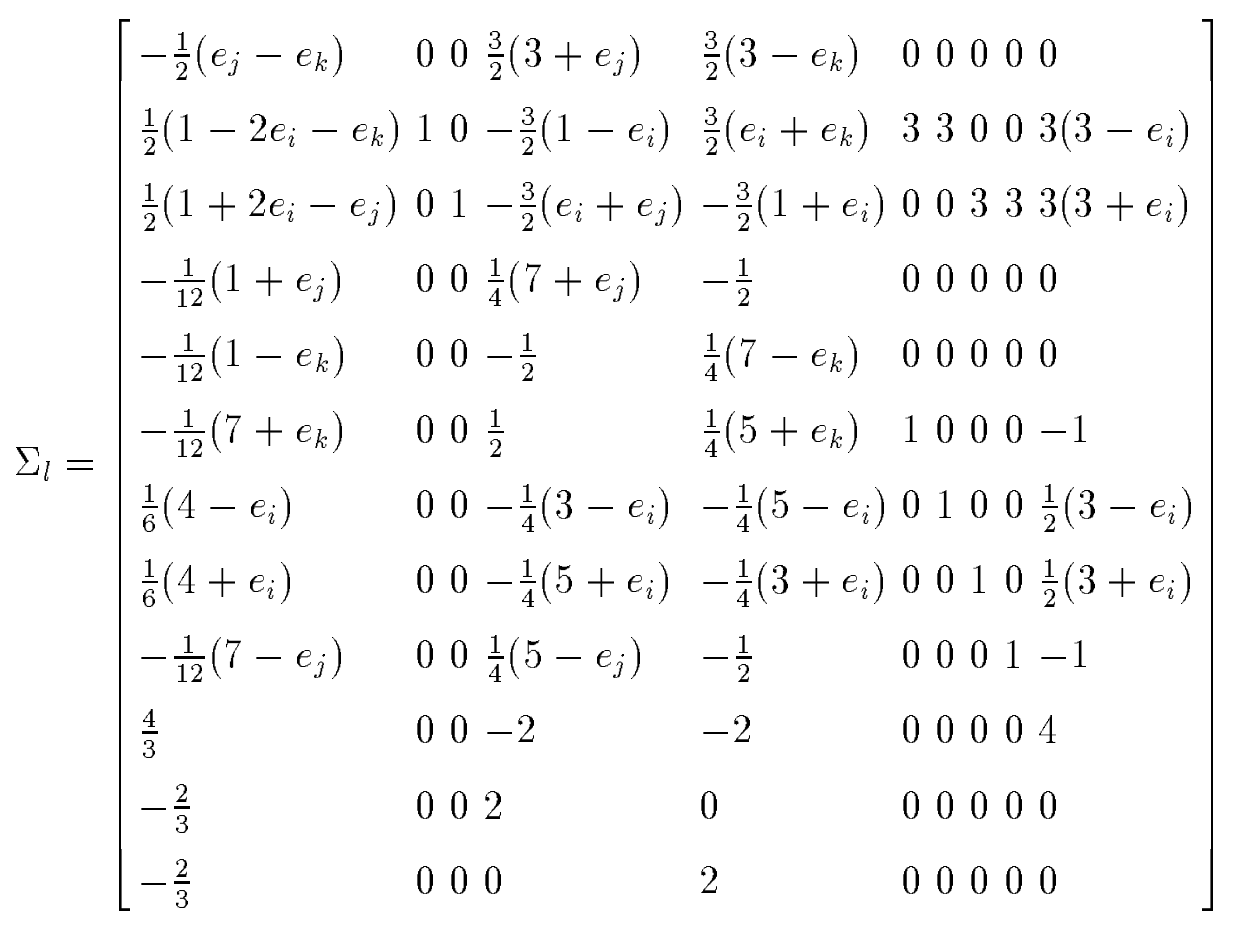

We have the equality $P_{T}=P_{3}$.

\subsubsection{The $C^{1}$ reduced cubic Hsieh-Clough-Tocher element (HCT-R)}

With the same notations as previously the set of degrees of freedom is given by

$$
\Sigma_{T}=\left\{f\left(a_{i}\right): 1 \leq i \leq 3 ; \partial_{x} f\left(a_{i}\right), \partial_{v} f\left(a_{i}\right): 1 \leq i \leq 3\right\} .
$$

The local interpolation operator $\Pi_{T}$ is determined for all $f$ in $C^{1}(\bar{T})$ by

$$
\Pi_{T} f_{\left.\right|_{K_{l}}}=\sum_{i=l}^{(l+1) \bmod 3+1} f\left(a_{i}\right) \Psi_{l, i}^{0}+\left(\nabla f\left(a_{i}\right) \cdot \overrightarrow{a_{i} a_{k}}\right) \Psi_{l, i, k}^{1}+\left(\nabla f\left(a_{i}\right) \cdot \overrightarrow{a_{i} a_{j}}\right) \Psi_{l, i, j}^{1}
$$

The basis functions $\left\{\Psi_{l}\right\}$ are defined by

$$
\Xi_{l}=\Sigma_{l} \Lambda_{l}
$$

with $i=l, j=i \bmod 3+1$ et $k=j \bmod 3+1$

$$
\Xi_{l}=\left(\Psi_{l, i}^{0}, \Psi_{l, j}^{0}, \Psi_{l, k}^{0}, \Psi_{l, i, k}^{1}, \Psi_{l, i, j}^{1}, \Psi_{l, j, i}^{1}, \Psi_{l, i, k}^{1} \Psi_{l, k, j}^{1}, \Psi_{l, k, i}^{1}\right)^{T},
$$




$$
\Lambda_{l}=\left(\lambda_{i}^{3}, \lambda_{j}^{3}, \lambda_{k}^{3}, \lambda_{i}^{2} \lambda_{k}, \lambda_{i}^{2} \lambda_{j}, \lambda_{j}^{2} \lambda_{i}, \lambda_{j}^{2} \lambda_{k}, \lambda_{k}^{2} \lambda_{j}, \lambda_{k}^{2} \lambda_{i}, \lambda_{i} \lambda_{j} \lambda_{k}\right)^{T}
$$

and

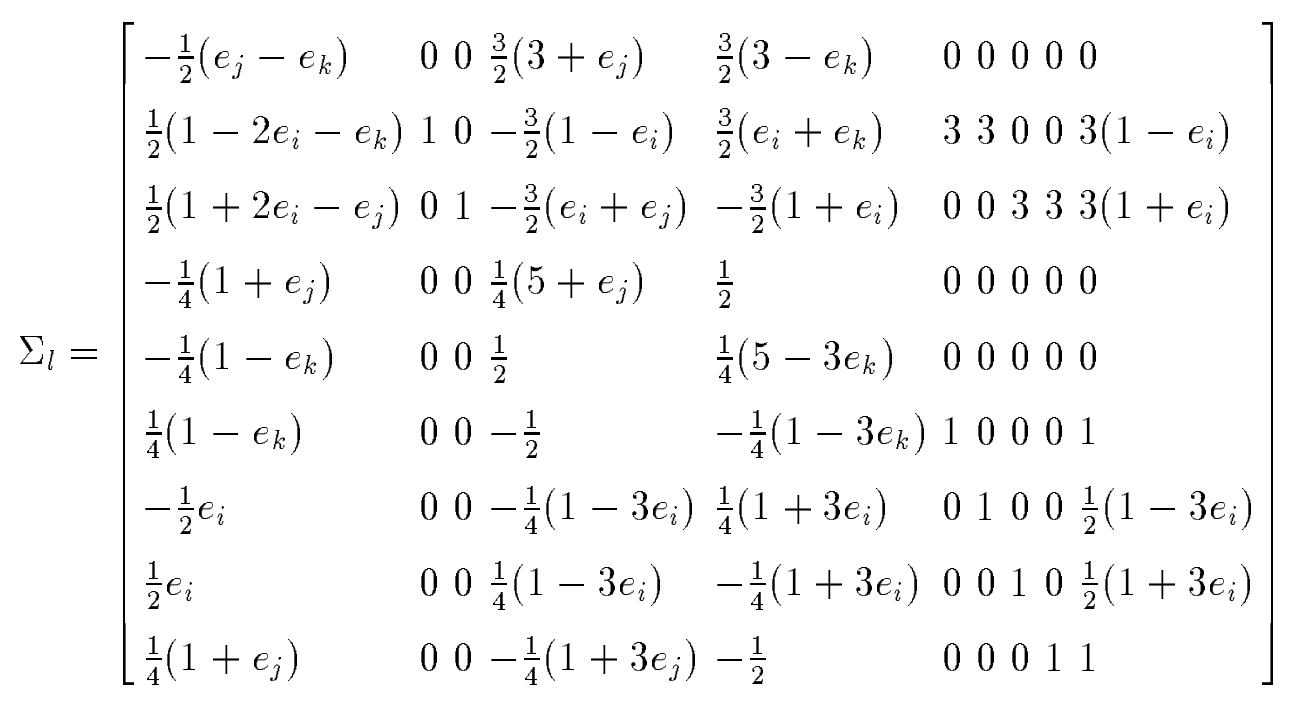

We have the relationship $P_{2} \subset P_{T} \subset P_{3}$.

\subsection{The fully discretized scheme}

Now, we can write the fully discretized scheme. From the local interpolation operator $\Pi_{T}$ we can define the global interpolation operator $\Pi_{h}$ from $C^{0}(\bar{\Omega})$ (resp. $C^{1}(\bar{\Omega})$ ) onto $X_{h}$ (resp. $Y_{h}$ ) by the relation (8). For example the discretization space $Y_{h}$ built on the $C^{1}$ cubic Nielson element admits basis functions $\left\{\varphi_{k}\right\}_{k=1, \ldots, N},\left\{\psi_{k}\right\}_{k=1, \ldots, N}$ and $\left\{\eta_{k}\right\}_{k=1, \ldots, N}$ such that

$$
\left(\Pi_{h} f\right)(x, v)=\sum_{k=1}^{N} f_{k} \eta_{k}(x, v)+\partial_{x} f_{k} \psi_{k}(x, v)+\partial_{v} f_{k} \varphi_{k}(x, v) \quad \forall f \in C^{1}(\bar{\Omega})
$$

where

$$
f_{k}=f\left(x_{k}, v_{k}\right), \quad \partial_{x} f_{k}=\left(\partial_{x} f\right)\left(x_{k}, v_{k}\right) \text { and } \partial_{v} f_{k}=\left(\partial_{v} f\right)\left(x_{k}, v_{k}\right)
$$

We start at the time $t^{n}$ where we know the discrete distribution function $f_{h}^{n}(x, v)$ that we can express as

$$
f_{h}^{n}(x, v)=\sum_{k=1}^{N} f_{h, k}^{n} \eta_{k}(x, v)+\partial_{x} f_{h, k}^{n} \psi_{k}(x, v)+\partial_{v} f_{h, k}^{n} \varphi_{k}(x, v),
$$

where the $\left\{f_{h, k}^{n}\right\}_{k=1}^{N},\left\{\partial_{x} f_{h, k}^{n}\right\}_{k=1}^{N}$ and $\left\{\partial_{v} f_{h, k}^{n}\right\}_{k=1}^{N}$ are the degrees of freedom of the space $Y_{h}$. Now we develop the algorithm to reach the time $t^{n+1}$. 
1. Perform a half time step advection in physical space:

$$
f_{h}^{\star}(x, v)=\Pi_{h} f_{h}^{n}(x-v \Delta t / 2, v),
$$

that is to say

$$
f_{h}^{\star}(x, v)=\sum_{k=1}^{N} f_{h, k}^{\star} \eta_{k}(x, v)+\partial_{x} f_{h, k}^{\star} \psi_{k}(x, v)+\partial_{v} f_{h, k}^{\star} \varphi_{k}(x, v)
$$

where

$$
\begin{aligned}
& f_{h, k}^{\star}=f_{h}^{n}\left(x_{k}-v_{k} \Delta t / 2, v_{k}\right) \\
& \partial_{x} f_{h, k}^{\star}=\partial_{x} f_{h}^{n}\left(x_{k}-v_{k} \Delta t / 2, v_{k}\right) \\
& \partial_{v} f_{h, k}^{\star}=-\frac{\Delta t}{2} \partial_{x} f_{h}^{n}\left(x_{k}-v_{k} \Delta t / 2, v_{k}\right)+\partial_{v} f_{h}^{n}\left(x_{k}-v_{k} \Delta t / 2, v_{k}\right) .
\end{aligned}
$$

2. Compute the electric field $E_{h}^{\star}(x)$ by substituting $f_{h}^{\star}$ in the Poisson equation which has to be discretized by an usual numerical scheme like finite difference or finite element method.

3. Perform a full timestep time advection in velocity space:

$$
f_{h}^{\star \star}(x, v)=\Pi_{h} f_{h}^{\star}\left(x, v-E_{h}^{\star}(x) \Delta t / 2, v\right),
$$

that is to say

$$
f_{h}^{\star \star}(x, v)=\sum_{k=1}^{N} f_{h, k}^{\star \star} \eta_{k}(x, v)+\partial_{x} f_{h, k}^{\star \star} \psi_{k}(x, v)+\partial_{v} f_{h, k}^{\star \star} \varphi_{k}(x, v),
$$

where

$$
\begin{aligned}
f_{h, k}^{\star \star} & =f_{h}^{\star}\left(x_{k}, v_{k}-E_{h}^{\star}\left(x_{k}\right) \Delta t\right) \\
\partial_{x} f_{h, k}^{\star \star} & =\partial_{x} f_{h}^{\star}\left(x_{k}, v_{k}-E_{h}^{\star}\left(x_{k}\right) \Delta t\right)-\Delta t \partial_{x} E_{h}^{\star}\left(x_{k}\right) \partial_{v} f_{h}^{\star}\left(x_{k}, v_{k}-E_{h}^{\star}\left(x_{k}\right) \Delta t\right) \\
\partial_{v} f_{h, k}^{\star \star} & =\partial_{v} f_{h}^{\star}\left(x_{k}, v_{k}-E_{h}^{\star}\left(x_{k}\right) \Delta t\right) .
\end{aligned}
$$

4. Perform a second half time step advection in physical space:

$$
f_{h}^{n+1}(x, v)=\Pi_{h} f_{h}^{\star \star}(x-v \Delta t / 2, v),
$$

that is to say

$$
f_{h}^{n+1}(x, v)=\sum_{k=1}^{N} f_{h, k}^{n+1} \eta_{k}(x, v)+\partial_{x} f_{h, k}^{n+1} \psi_{k}(x, v)+\partial_{v} f_{h, k}^{n+1} \varphi_{k}(x, v),
$$


where

$$
\begin{aligned}
& f_{h, k}^{n+1}=f_{h}^{\star \star}\left(x_{k}-v_{k} \Delta t / 2, v_{k}\right) \\
& \partial_{x} f_{h, k}^{n+1}=\partial_{x} f_{h}^{\star \star}\left(x_{k}-v_{k} \Delta t / 2, v_{k}\right) \\
& \partial_{v} f_{h, k}^{n+1}=-\frac{\Delta t}{2} \partial_{x} f_{h}^{\star \star}\left(x_{k}-v_{k} \Delta t / 2, v_{k}\right)+\partial_{v} f_{h}^{\star \star}\left(x_{k}-v_{k} \Delta t / 2, v_{k}\right) .
\end{aligned}
$$

\subsection{Positive and conservative schemes}

The previous schemes do not preserve positivity and mass conservation mass. Indeed this inconvenient comes from the interpolation operator. The non preservation of positivity and mass conservation could be an inconvenient for lengthy simulations since numerical oscillations develop. In order to recover positivity and mass conservation we use the ideas of Priestley [17] and Staniforth [19] which have been applied in numerical weather prediction codes.

First we are going to define the properties, we expect the solution to verify. The algorithm provides a positive solution if the solution verifies the following maximum principle :

$$
f_{\min }^{n} \leq f_{h, l}^{n+1} \leq f_{\max }^{n}, \quad \forall l, \forall n
$$

where

$$
f_{\min }^{n}=\min _{k}\left\{f_{h, k}^{n}\right\}, \quad f_{\max }^{n}=\max _{k}\left\{f_{h, k}^{n}\right\} .
$$

The solution conserves the mass if

$$
\sum_{k} f_{h, k}^{n} \mathcal{A}_{k}=\sum_{k} f_{h, k}^{0} \mathcal{A}_{k}=M_{0}
$$

where $\mathcal{A}_{k}$ is the area associated to the node $N_{k}$ such that $\cup_{k} \mathcal{A}_{k}=\bar{\Omega}$.

Now we explain how to obtain a positive and conservative solution by applying it on the first half advection in the physical space. Note that the algorithm will be the same for the other advections.

Let $f_{L_{h}}^{\star}(x, v)$ a low order solution computed according to the first step of the algorithm described previously, with the Lagrange interpolation operator of order one for example. Let $f_{H_{h}}^{\star}(x, v)$ a high order solution computed according to the first step of the algorithm described previously, with one of Hermite interpolation operator we see before. Let $f_{L_{h}, k}^{\star}=f_{L_{h}}^{\star}\left(x_{k}, v_{k}\right)$ and $f_{H_{h}, k}^{\star}=f_{H_{h}}^{\star}\left(x_{k}, v_{k}\right)$. We suppose that the triangle $T_{j}$ contains the point $\left(x_{k}-\right.$ $\left.v_{k} \Delta t / 2, v_{k}\right)$ which is at the time $t^{n}$ the departure point approximation of the characteristic curve ending at the point $\left(x_{k}, v_{k}\right)$ at the time $t^{n+1}$. Let $N_{T}$ the number of nodes carried by a triangle $T$ on which we know $f_{h}^{n}$. We define $f^{+}$ and $f^{-}$by

$$
f^{+}=\max \left\{f_{h, 1}^{n}, \ldots, f_{h, N_{T}}^{n}\right\}
$$


and

$$
f^{-}=\min \left\{f_{h, 1}^{n}, \ldots, f_{h, N_{T}}^{n}\right\}
$$

Then we set

$$
\bar{f}_{k}=\left\{\begin{array}{l}
f^{+} \text {if } f_{H_{h}, k}^{\star}>f^{+} \\
f^{-} \text {if } f_{H_{h}, k}^{\star}<f^{-} \\
f_{H_{h}, k}^{\star} \text { otherwise. }
\end{array}\right.
$$

If $f_{L_{h}, k}^{\star}-f_{H_{h}, k}^{\star} \neq 0$ then we define

$$
\gamma_{k}^{\max }=\frac{\bar{f}_{k}-f_{L_{h}, k}^{\star}}{f_{H_{h}, k}^{\star}-f_{L_{h}, k}^{\star}},
$$

otherwise we set

$$
\gamma_{k}^{\max }=1
$$

Then, if we set

$$
f_{h, k}^{\star}=\gamma_{k}^{\max } f_{H_{h}, k}^{\star}+\left(1-\gamma_{k}^{\max }\right) f_{L_{h}, k}^{\star}
$$

we see that the solution satisfies the maximum principle in sense of (9). In order to have a conservative solution we have to replace the set $\left\{\gamma_{k}^{\max }\right\}$ by the set of optimal $\left\{\gamma_{k}\right\}$ for which the solution still preserves the maximum principle and the mass conservation. In order to get a solution which preserves the maximum principle (9) the set $\left\{\gamma_{k}\right\}$ must satisfy the constraints

$$
0 \leq \gamma_{k} \leq \gamma_{k}^{\max }
$$

Moreover, if we want that the solution preserves mass conservation we impose the constraint (10). Let us define

$$
\xi_{k}=\left(f_{H_{h}, k}^{\star}-f_{L_{h}, k}^{\star}\right) \mathcal{A}_{k}
$$

then the condition (10) can be rewritten as

$$
\sum_{k} \gamma_{k} \xi_{k}=M_{0}-\sum_{k} f_{L_{h}, k}^{\star} \mathcal{A}_{k}=M^{\star}
$$

Then the problem can consist in minimizing

$$
-\sum_{k} \gamma_{k}
$$

where the unknowns $\left\{\gamma_{k}\right\}$ are subject to the constraints

$$
0 \leq \gamma_{k} \leq \gamma_{k}^{\max }, \quad \sum_{k} \gamma_{k} \xi_{k}=M^{\star}
$$

This kind of problems can be solved by linear programming methods as the simplex method. Next we present a direct way of obtaining a solution. Note 
that to enforce the conservation of other physical quantities such that energy, entropy or high order moments we can extend this method which leads to linear or non linear optimization problems with constraints. This method can also be used to minimize the the loss of preserved quantities like the $L^{2}$ norm. First, if we have

$$
\sum_{k} \gamma_{k}^{\max } \xi_{k}=M^{\star}
$$

then the mass is conserved and we have found a postive solution which preserves the discrete maximum principle (9) and the mass conservation (10).

Now assume that

$$
\sum_{k} \gamma_{k}^{\max } \xi_{k}>M^{\star}
$$

If it is not the case, without loss of generality, we put $\xi_{k}=-\xi_{k}$ and $M^{\star}=-M^{\star}$ so as to achieve the inequality (12).

In order to reduce as much as possible the size of the left hand size of (12) the negative and zero terms of the sums (12) are supplied with highest coefficient, that is to say

$$
\begin{aligned}
& \text { if } \xi_{k} \leq 0 \text { then set } \gamma_{k}=\gamma_{k}^{\max }, \operatorname{iflag}(k)=1, \\
& \text { if } \xi_{k}>0 \text { then set } \gamma_{k}=0, \text { iflag }(k)=0
\end{aligned}
$$

Now we have to compute the coefficients $\gamma_{k}$ for which iflag $(k)=0$. To do this we define a surplus as

$$
\text { surplus }=M^{\star}-\sum_{i f l a g(k)=1} \gamma_{k} \xi_{k}
$$

and an average value of $\gamma_{k}$ as

$$
\gamma_{a v}=\frac{\text { surplus }}{\sum_{i f l a g(k)=0} \gamma_{k} \xi_{k}} .
$$

Note that the surplus or all $\xi_{k}$ can be negative. In that case there is no conservative solution and the best thing to do to limit as much as possible the loss of mass is to set $\gamma_{k}$ as it was done for the initial set-up (13). In other case all the values $\gamma_{k}^{\max }$ for which iflag $(k)=0$ are compared with the average value $\gamma_{a v}$ and set equal to the average if it does not exceed all the upper bounds, that is to say

$$
\text { if } \gamma_{a v}<\gamma_{k}^{\max } \forall k \text { such that iflag }(k)=0 \text {, then set } \gamma_{k}=\gamma_{a v} \text {, iflag }(k)=1 \text {. }
$$

On the other hand, if some coefficients $\gamma_{k}^{\max }$ for which iflag $(k)=0$ are smaller than the average value $\gamma_{a v}$, then those coefficients are set equal to their maximum value :

$$
\text { if } \gamma_{a v}>\gamma_{k}^{\max } \forall k \text { such that iflag }(k)=0 \text {, then set } \gamma_{k}=\gamma_{k}^{\max } \text {, iflag }(k)=1 \text {. }
$$


The other coefficients are left with $i f \operatorname{lag}(k)=0$. Then a new evaluation of (14) and (15) is performed. The algorithm finish successfully when we found a value $\gamma_{a v}$ which does not exceed any $\gamma_{k}^{\max }$ or when $\operatorname{iflag}(k)=1$ for all $k$. Note that seeking optimal $\left\{\gamma_{k}\right\}$ to enforce mass conservation will sacrifice results accuracy.

\section{Numerical results}

In this section we present numerical results obtained in plasma physics and in the propagation of charged particle beams.

\subsection{The 2D Vlasov-Poisson system in phase space}

In this section we compare different methods conservative or not, with different reconstructive techniques for the numerical resolution of the Vlasov-Poisson system with periodic boundary conditions

$$
\frac{\partial f}{\partial t}+v \frac{\partial f}{\partial x}+E(t, x) \frac{\partial f}{\partial v}=0
$$

coupled with the normalized Poisson equation

$$
E(t, x)=-\frac{\partial \phi}{\partial x}, \quad-\frac{\partial^{2} \phi}{\partial x^{2}}=\int_{\mathbb{R}} f(t, x, v) d v-1 .
$$

According to the numerical scheme described in section 3 we have to compute the gradient of the electric field. Here we this calculus is easy because we see that

$$
\frac{\partial E}{\partial x}(t, x)=\rho(x)-1=\int_{\mathbb{R}} f(t, x, v) d v-1 .
$$

\subsubsection{The linear Landau damping}

The initial data are

$$
f(0, x, v)=\frac{1}{\sqrt{2 \pi}} \exp \left(-v^{2} / 2\right)(1+\alpha \cos (k x)), \quad \forall(x, v) \in[0, L] \times \mathbb{R},
$$

where $\alpha=0.01$ stands for intensity of the perturbation. The length period is $L=4 \pi$ and $v^{\max }=6$ is the value beyond which we consider the distribution function is equal to zero. The time step $\Delta t=1 / 8$ and $N_{\text {mesh }}$ represents the number of mesh cell. The final time $T=50 \omega_{p}^{-1}$.

The figure 1 shows the comparison of the evolution of the first mode $(k=0.5)$ 
of the electric filed, for different interpolation operators. Here the cell number is 3906 and we do neither enforce the positivity and the mass conservation of the solution. The amplitude of the first mode $E(k=0.5, t)$ decreases exponentially as it is predicted by the theory. The damping rate and the frequency of oscillations obtained by these methods are respectively given by $\gamma=0.153$ and $\omega=1.415$, which agree very well with values $\gamma=0.1533$ and $\omega=1.4156$ predicted by the theory. For this physical case the differences between the methods appear in long time. The best schemes are those that use $C^{1}$ reconstructions and the less satisfactory is the Lagrange operator of order 2 . On the figure 3 (a) we observe that the "recurrence effect" (see [14]) appears at $T_{R}=33.49$, which is the theoretical time predicted from the free streaming case (i.e. without electric field) since $T_{R}=2 \pi /(k \Delta v)$. Although there is no conservation enforcing we observe that the distribution function remains positive and the relative error norm of variations of kinetic entropy, mass, $L^{1}$ norm, $L^{2}$-norm and total energy always stay less than $10^{-5}$. The figures 3 et 2 compare for different meshes, the effectiveness of the interpolation operators and the effect of the positivity and the mass conservation enforcing on the first mode. First NC1 et HCT-C interpolation operators give very similarly results. In the conservative case we observe that the evolution of the first mode in long time is less accurate but when we refine the mesh the approximation of the solution is improved. Then the algorithm seems to converge.
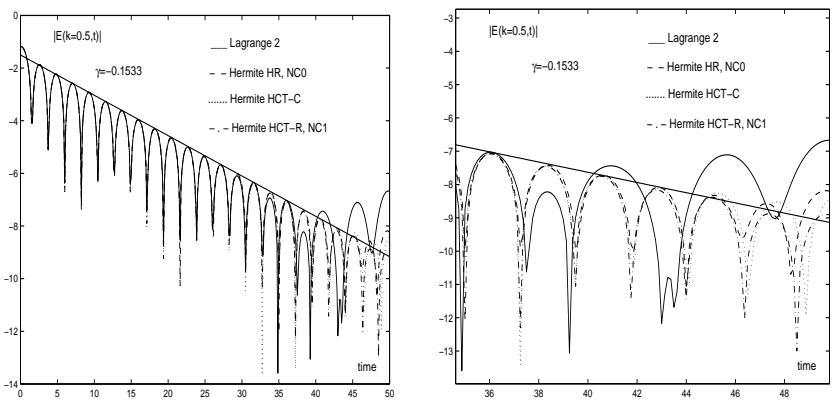

Fig. 1. Comparison of interpolation operators on the evolution of the first mode $E(k=0.5, t)$ of the electric field for the linear Landau damping. $N_{\text {mesh }}=3906$.

\subsubsection{The strong Landau damping}

The initial data are

$$
f(0, x, v)=\frac{1}{\sqrt{2 \pi}} \exp \left(-v^{2} / 2\right)(1+\alpha \cos (k x)), \quad \forall(x, v) \in[0, L] \times \mathbb{R},
$$

where the amplitude of the initial perturbation of the density is increased. We take $\alpha=0.5$. We still take $L=4 \pi, k=0.5, \Delta t=1 / 8$ and $v^{\max }=6$. The number of cell is $N_{\text {mesh }}=2048$. The electric field introduce a strong modulation of the charge density. The previous theory can not be applied as 


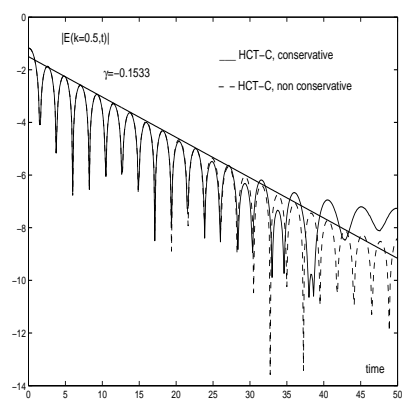

(a) $N_{m e s h}=3906$

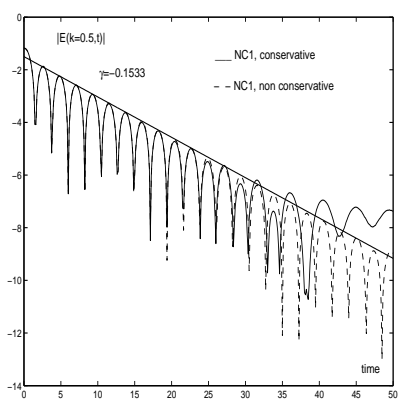

(b) $N_{m e s h}=3906$

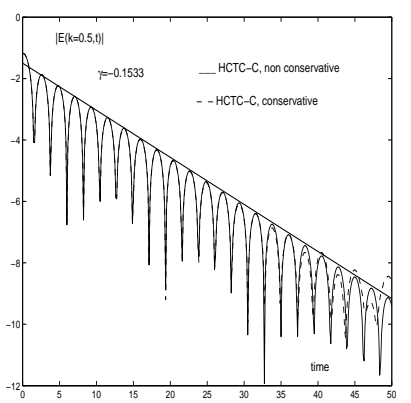

(c) $N_{m e s h}=7874$

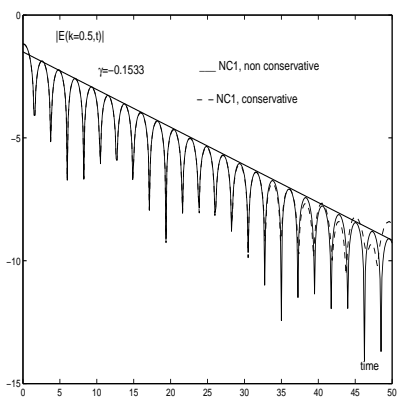

(d) $N_{m e s h}=7874$

Fig. 2. Evolution of the first mode $E(k=0.5, t)$ of the electric field for the linear Landau damping.

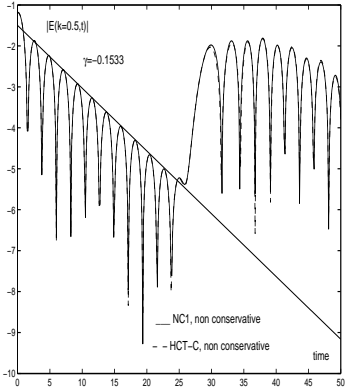

(a) $N_{m e s h}=1092$

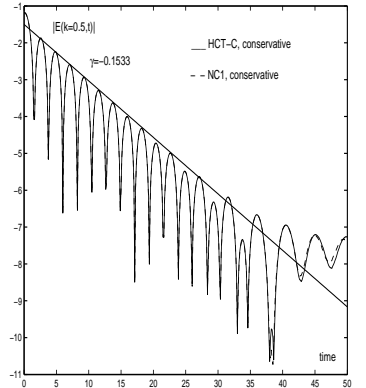

(b) $N_{m e s h}=3906$

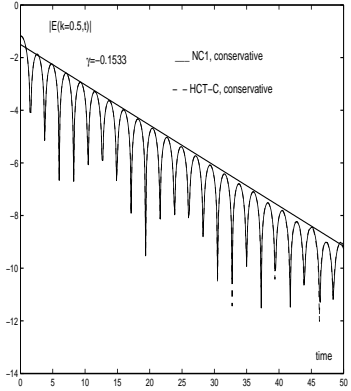

(c)

Fig. 3. Evolution of the first mode $E(k=0.5, t)$ of the electric field for the linear Landau damping.

non linear effects are too important but this test has been studied by many authors ([11], [9], [14], [16],[5]). The amplitude of the first mode decreases exponentially in a first time, and oscillate periodically around a constant in a second time. First the electric waves give energy to the particles, that's why we observe the exponential decrease of the first mode which contains the most 
of the part of electric energy. Then, after the initial condition mixing and the transitory phenomena, particles whose kinetic energy is smaller than the potential energy, are trapped by electrostatic waves around the phase velocity $v_{\phi}=\omega / k$, where small bumps appear preceded by small holes (10). The deeply trapped electrons oscillate in the wave potential with a bounce frequency which is the frequency of the envelope of the first mode (see [7], [4], [10], [13]). When the algorithm is non conservative we observe (Fig. 4,5) that with Lagrange interpolation of order two the preservation of the $L^{1}$ norm is better on short time but the scheme is to dissipative (important loss of the $L^{2}$ norm and entropy). The Hermite $C^{1}$ type interpolation (HCT-C, HCT-R, NC1) gives good results which are similar even better in term of conservation than results obtained by cubic spline on Cartesian grid (see [9]). We observe a loss of mass less than $1.16 \%$ during the transitory phenomena (strong variations of the distribution function caused by non linear effects) which is reduced in long time to a relative error of order $10^{-3}$. We observe a loss of the $L^{2}$ norm of $10 \%$ and a growth of the entropy of $16 \%$ which stabilize in long time. Among the $C^{1}$ interpolation techniques the best interpolation operator in term of $L^{1}$ norm conservation are HCT-R and NC1, and in term of conservation of the $L^{2}$ norm (the less diffusive) the HCT-C interpolation operator is the best. On the contrary the Lagrange interpolation of order two is twice more diffusive. The conservative version of the algorithm does not change the results described previously except that now the solution is positive, and preserves the mass conservation (also $L^{1}$ norm) and the maximum principle. These conservations are useful for lengthy simulations since numerical oscillations can develop when the non linear effects are dominating. Indeed if we do not search to stabilize or control the growth of these oscillations the scheme can become numerically unstable and blow up. That is what occurs with Lagrange interpolation of order 3 or more when we apply the non conservative version of the algorithm. Moreover the decrease of the physical entropy and the $L^{2}$ norm is associated to the fact that the distribution function is smoothed when filaments become smaller than the phase space cell size. Indeed in the semi-Lagrangian method the projection onto the mesh means that we solve the Vlasov equation at the scale of the size of the phase space cell. Then the development of thin structures is not taken into account by the scheme and the physic phenomena of high frequency are not reproduced. The projection onto the mesh plays the part of a low-pass filter.

\subsubsection{The two stream instability}

The initial condition are

$$
f(0, x, v)=\frac{1}{\sqrt{2 \pi}} v^{2} \exp \left(-v^{2} / 2\right)(1+\alpha \cos (k x)), \quad \forall(x, v) \in[0, L] \times \mathbb{R},
$$




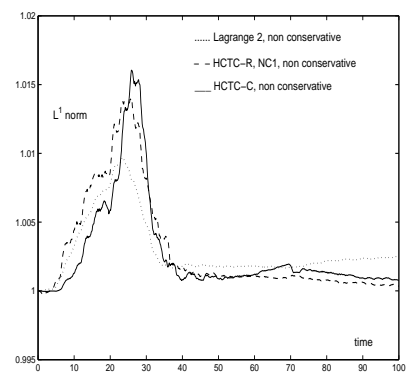

Fig. 4. Evolution of the $L^{1}$ norm for the strong non linear Landau damping. $N_{\text {mesh }}$ $=3906$.

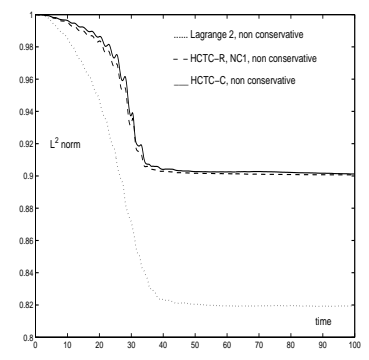

(a) $L^{2}$ norm

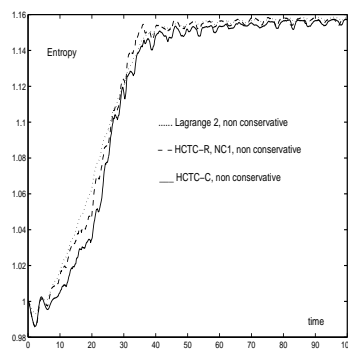

(b) Entropy

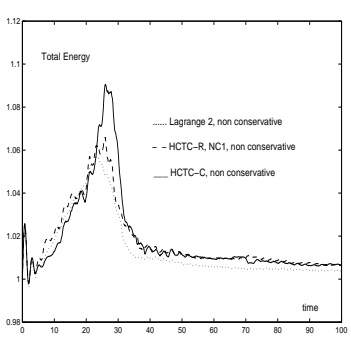

(c) Total energy

Fig. 5. Evolution of the $L^{2}$ norm, entropy and total energy for the strong non linear Landau damping in the case of the non conservative algorithm. $N_{m e s h}=3906$

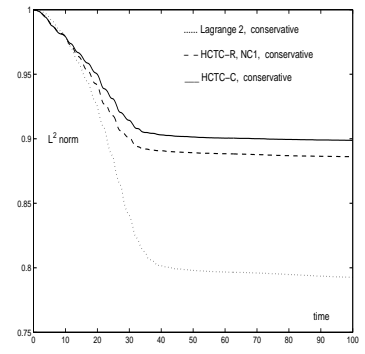

(a) $L^{2}$ norm

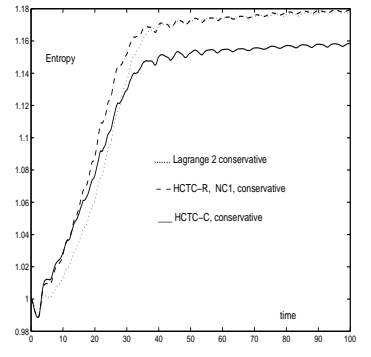

(b) Entropy

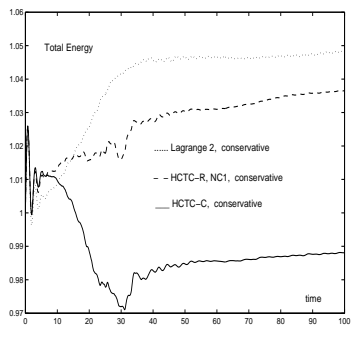

(c) Total energy

Fig. 6. Evolution of the $L^{2}$ norm, entropy and total energy for the strong non linear Landau damping in the case of the conservative algorithm. $N_{\text {mesh }}=3906$

where $\alpha=0.05, L=4 \pi$ and $v^{\max }=6$. The time step $\Delta t$ is equal to $1 / 8$ and $N_{\text {mesh }}=3096$. The final time $T$ is $100 \omega_{p}^{-1}$.

The figures 18 shows the time evolution of the distribution function in the phase space. At time $t \cong 10$, we observe the formation of a vortex which is associated to trapped particles. From $t \cong 10$ until $t \cong 20$, the instability grows rapidly and a hole appears. After $t \cong 20$ the trapped particles oscillate in the electrostatic potential and the vortex rotates periodically. The figures 15 and 


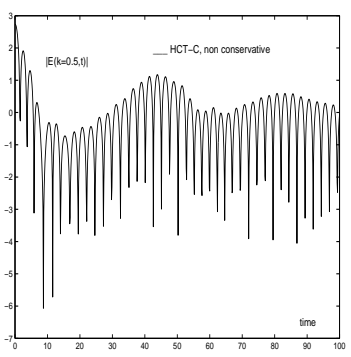

(a) HCT-C, non conservative

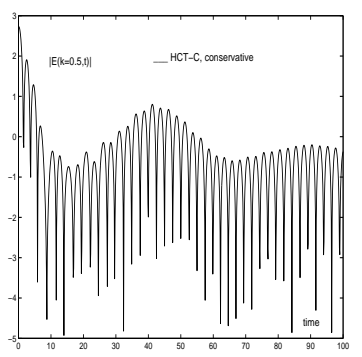

(b) HCT-C, conservative

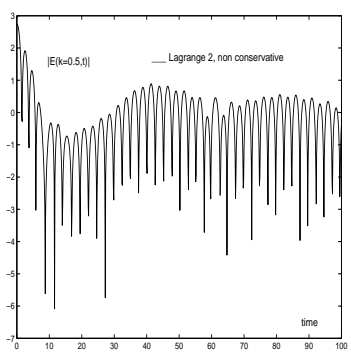

(c) Lagrange 2, non conservative

Fig. 7. Evolution of the first mode $E(k=0.5, t)$ of the electric field for the strong non linear Landau damping. $N_{m e s h}=3906$

Fig. 8 .

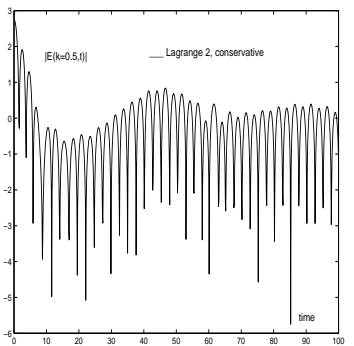

(a) Lagrange 2, conservative

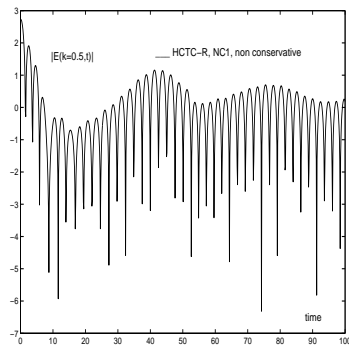

(b) $\mathrm{NC} 1, \mathrm{HCT}-$

$\mathrm{R}$, non conservative

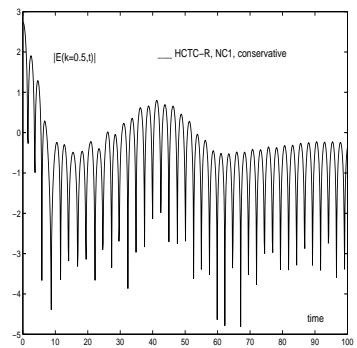

(c) $\mathrm{NC} 1, \mathrm{HCT}-$

$\mathrm{R}$, conservative

Fig. 9. Evolution of the first mode $E(k=0.5, t)$ of the electric field for the strong non linear Landau damping. $N_{\text {mesh }}=3906$

16 illustrate the evolution of the first three modes $E(k=0.5, t), E(k=1, t)$ and $E(k=1.5, t)$ according to the different interpolation operators and the conservative or non conservative version of the algorithm. After an initial transitory phase the first mode $E(k=0.5, t)$ increases exponentially to reach its maximum at the time $t \cong 18$, then it stabilizes and oscillates slowly because of particles trapping. The two other modes $E(k=1, t)$ et $E(k=1.5, t)$ increase exponentially and oscillate but always stay under the first mode. The electric energy increases rapidly from $t \cong 10$ to $t \cong 20$, and then oscillates periodically. We notice that the scheme with Lagrange interpolation of order two becomes bad in long time when we do not enforce the maximum principle and the mass conservation. In the case of the algorithm preserves the maximum principle and the mass conservation the Lagrange scheme of order two gives right results even if it remains more dissipative than the other schemes. The scheme of $C^{1}$ Hermite type (NC1, HCT-R, HCT-C) give good results. However we observe that HCT-C scheme is better in term of conservation of the entropy, 


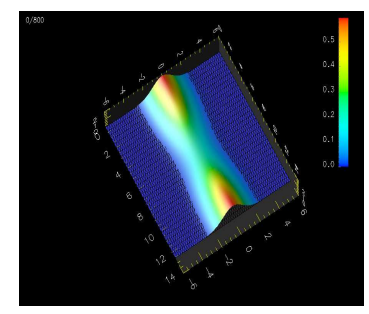

(a) $t=0 \omega_{p}^{-1}$

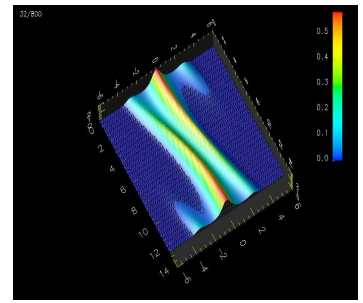

(d) $t=4 \omega_{p}^{-1}$

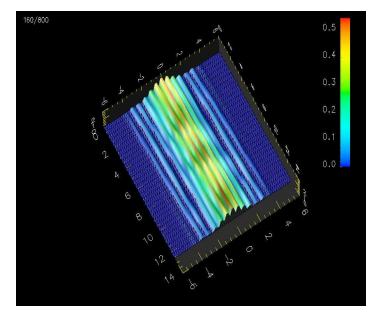

(g) $t=20 \omega_{p}^{-1}$

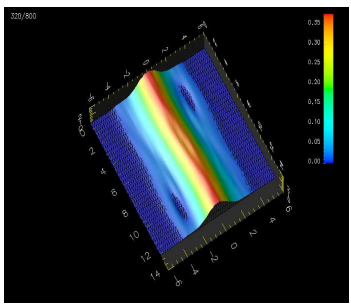

(j) $t=40 \omega_{p}^{-1}$

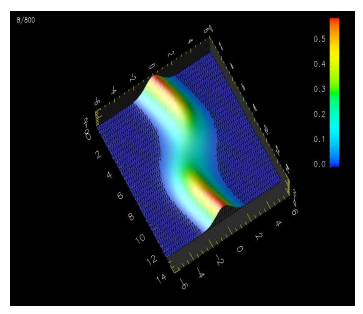

(b) $t=1 \omega_{p}^{-1}$

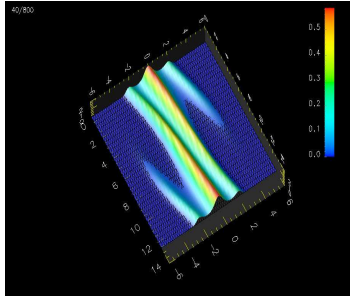

(e) $t=5 \omega_{p}^{-1}$

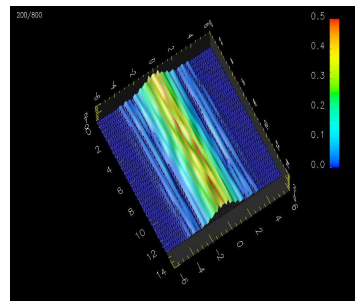

(h) $t=25 \omega_{p}^{-1}$

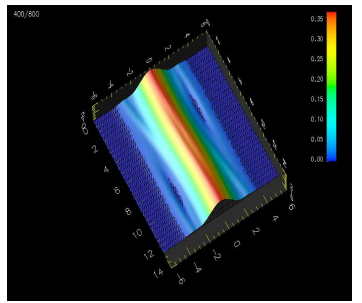

(k) $t=50 \omega_{p}^{-1}$

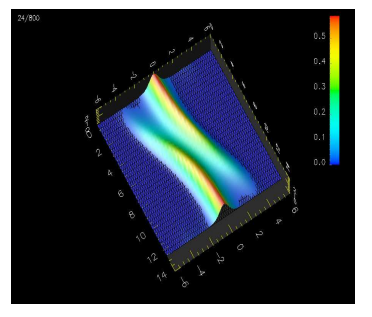

(c) $t=3 \omega_{p}^{-1}$

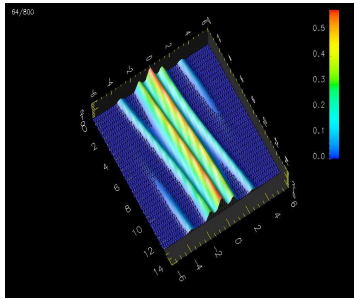

(f) $t=8 \omega_{p}^{-1}$

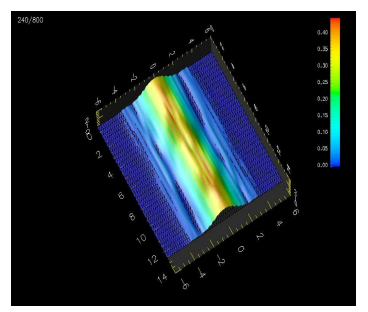

(i) $t=30 \omega_{p}^{-1}$

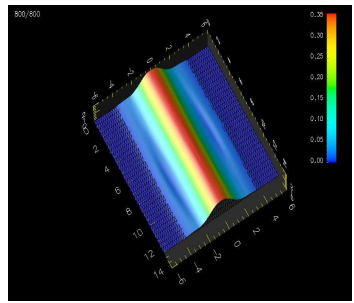

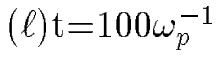

Fig. 10. Evolution of the distribution function $f$ in phase space for the NC1 conservative scheme $N_{m e s h}=3906$, in the case of the strong non linear Landau damping.

$L^{2}$ norm and energy. Moreover we note that the filamentation develops from $t \cong 15$ until $t \cong 35$, and then is smoothed in long time. The same numerical phenomenon of filtering explained for the strong non linear Landau damping occurs here yet. 

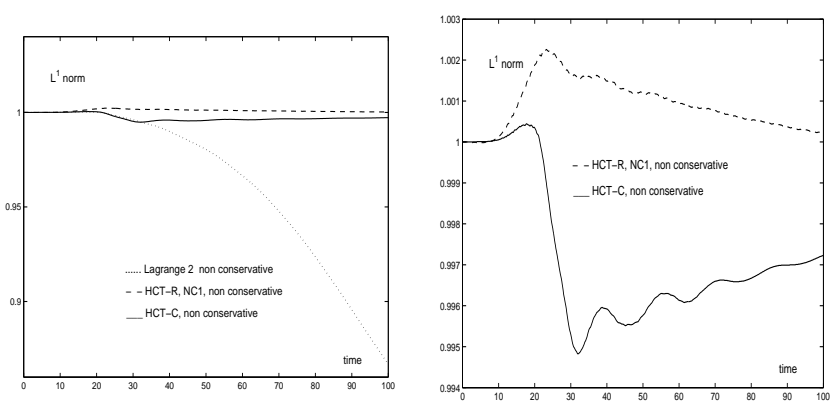

Fig. 11. Comparison of the interpolation operator on the evolution of the $L^{1}$ norm with the non conservative scheme in the case of two stream instability. $N_{\text {mesh }}=$ 3906 .

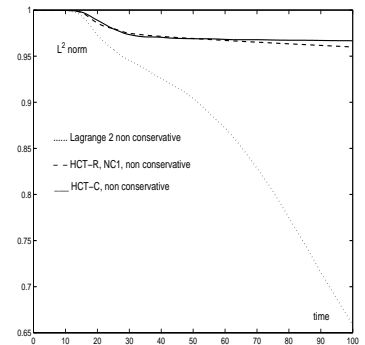

(a) $L^{2}$ norm

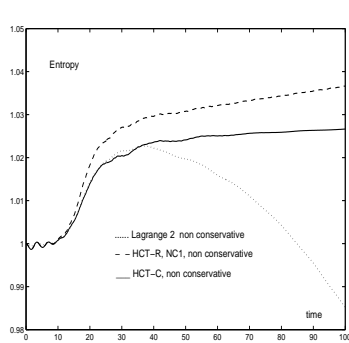

(b) Entropy

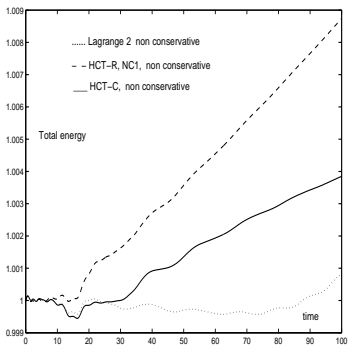

(c) Total energy

Fig. 12. Evolution of the $L^{2}$ norm, entropy and total energy, with the non conservative scheme in the case of two stream instability. $N_{\text {mesh }}=3906$

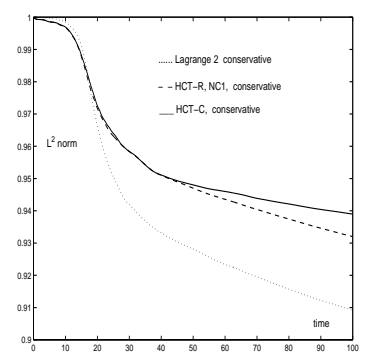

(a) $L^{2}$ norm

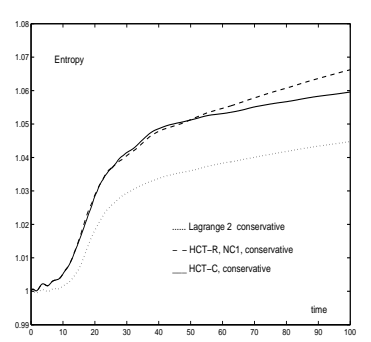

(b) Entropy

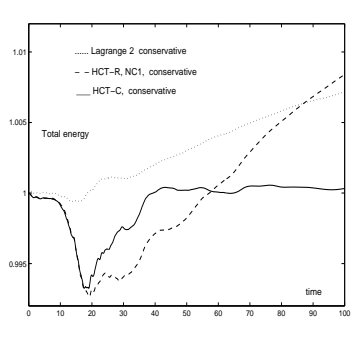

(c) Total energy

Fig. 13. Evolution of the $L^{2}$ norm, entropy and total energy, with the conservative scheme in the case of two stream instability. $N_{\text {mesh }}=3906$

\subsection{The axisymetric Vlasov-Poisson system}

The axisymetric Vlasov-Poisson system is very useful in the study of the propagation of charged particles beams. Here we suppose that the beam is uniform in the longitudinal variable $z$ and propagates with constant velocity $v_{z}$. The transverse component $\left(v_{x}, v_{y}\right)$ are small compared to the longitudinal velocity 


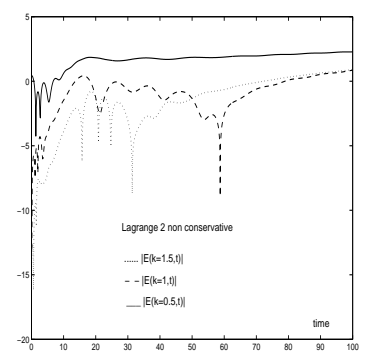

(a) Lagrange 2, non conservative

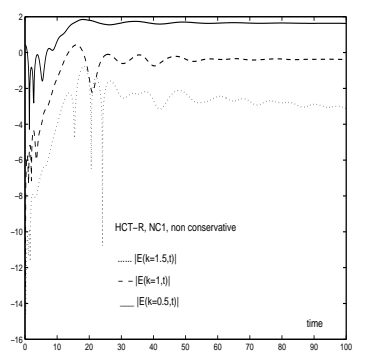

(b) $\mathrm{NC} 1, \mathrm{HCT}-$

$\mathrm{R}$, non conservative

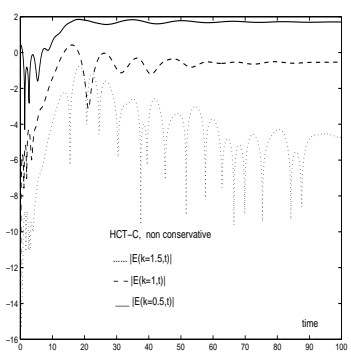

(c) HCT-C, non conservative

Fig. 14. Evolution of the first three modes $E(k=0.5, t), E(k=1, t)$ and $E(k=1.5, t)$ of the electric field with the non conservative algorithm in the case of the two stream instability. $N_{\text {mesh }}=3906$

Fig. 15 .

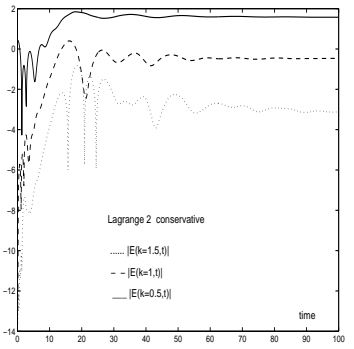

(a) Lagrange 2, conservative

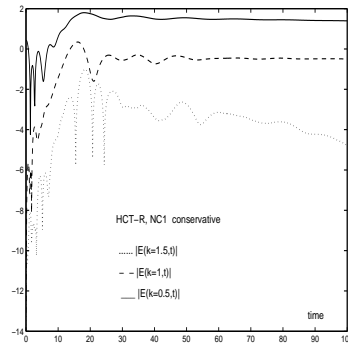

(b) $\mathrm{NC} 1, \mathrm{HCT}-$

$\mathrm{R}$, conservative

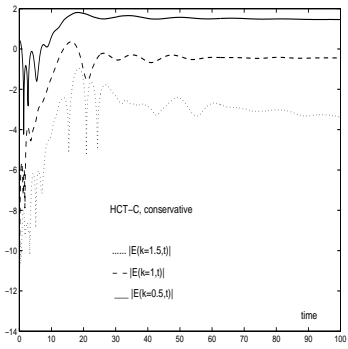

(c) HCT-C, conservative

Fig. 16. Evolution of the first three modes $E(k=0.5, t), E(k=1, t)$ and $E(k=1.5, t)$ of the electric field with the conservative algorithm in the case of the two stream instability. $N_{\text {mesh }}=3906$

$v_{z}$. The problem is then reduced to study the classical Vlasov equation in the transverse space. Moreover we suppose that the initial data are invariant by rotation. Then the solution of the Vlasov equation still remains invariant by rotation and satisfies the following equation written in dimensionless cylindrical variables

$$
\frac{\partial f}{\partial t}+v_{r} \frac{\partial f}{\partial r}+\left(E_{s}(t, r)+E_{a}(r, t)+\frac{v_{\theta}^{2}}{r}\right) \frac{\partial f}{\partial v_{r}}-\frac{v_{\theta} v_{r}}{r} \frac{\partial f}{v_{\theta}}=0
$$

where the radial electric field $E_{s}(t, r)$ is given by the Poisson equation

$$
\frac{1}{r} \frac{\partial\left(r E_{s}\right)}{\partial r}=\rho(t, r), \quad \rho(t, r)=\int_{\mathbf{R}^{2}} f\left(t, r, v_{r}, v_{\theta}\right) d v_{r} d v_{\theta}
$$




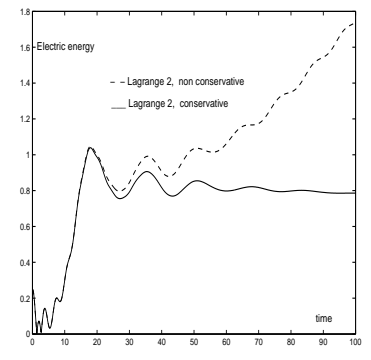

(a) Lagrange 2

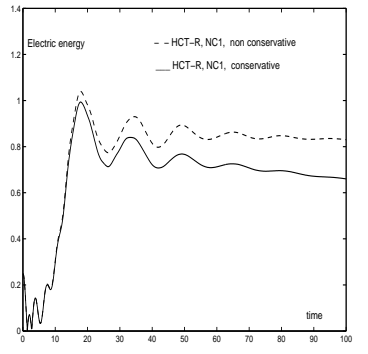

(b) NC1, HCT-

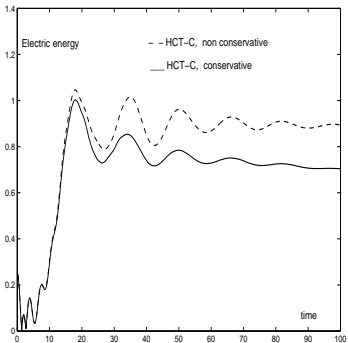

(c) HCT-C $\mathrm{R}$

Fig. 17. Comparison of the evolution of electric energy in the case of the two stream instability. $N_{m e s h}=3906$

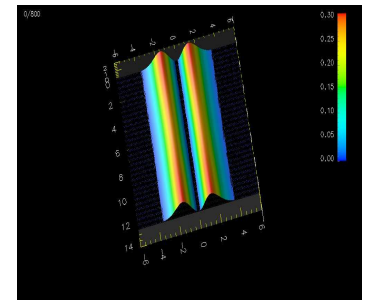

(a) $t=0 \omega_{p}^{-1}$

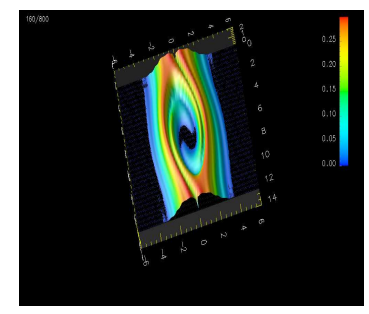

(d) $t=20 \omega_{p}^{-1}$

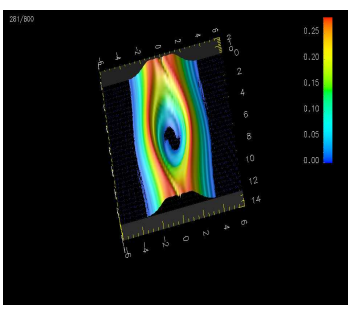

(g) $t=35 \omega_{p}^{-1}$

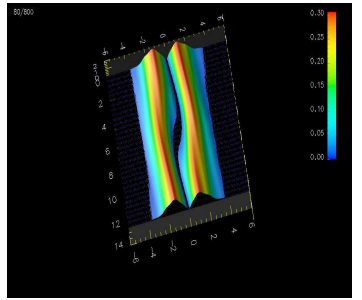

(b) $t=10 \omega_{p}^{-1}$

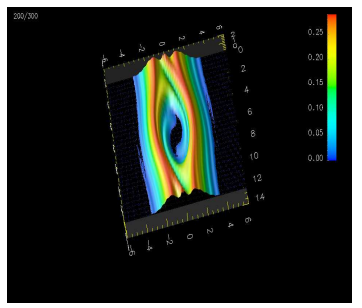

(e) $t=25 \omega_{p}^{-1}$

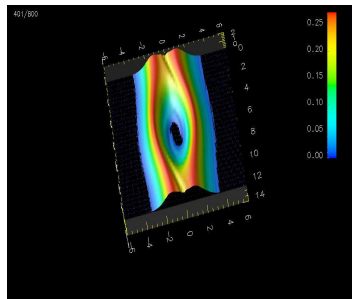

(h) $t=50 \omega_{p}^{-1}$

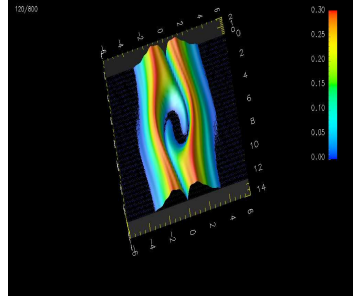

(c) $t=15 \omega_{p}^{-1}$

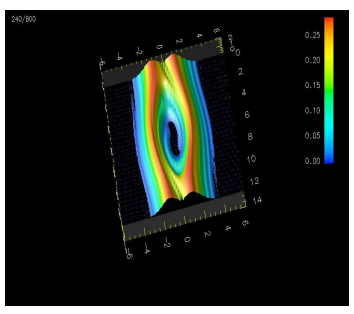

(f) $t=30 \omega_{p}^{-1}$

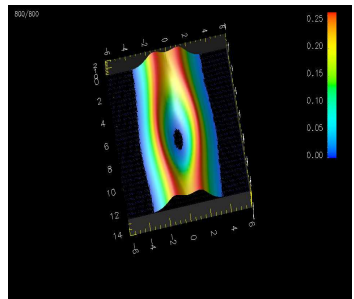

(i) $t=100 \omega_{p}^{-1}$

Fig. 18. Evolution of distribution function $f$ in the phase space, with the conservative NC1 scheme and $N_{\text {mesh }}=16320$, in the case of the two stream instability. 
and where the applied radial electric field $E_{a}(t, r)$ is supposed to be linear and equal to $-\omega_{0}^{2} r$.

If we make the variables change $\left(r, v_{\theta}, v_{r}\right) \longrightarrow\left(r, v_{r}, I\right)$ where $I=r v_{\theta}$ is the kinetic momentum in the $\mathrm{z}$-direction, the equation (16) becomes

$$
\frac{\partial f}{\partial t}+v_{r} \frac{\partial f}{\partial r}+\left(E_{s}(t, r)+\frac{I^{2}}{r^{3}}-\omega_{0}^{2} r\right) \frac{\partial f}{\partial v_{r}}=0 \quad \forall I \in \mathbb{R} .
$$

This formulation is well suited for parallelization as the variable $I$ plays the role of a parameter.

The Kapchinsky-Vladirmisky (K-V) distribution

$$
f_{0}\left(r, v_{r}, v_{\theta}\right)=\frac{n_{0}}{\pi} \delta_{0}\left(2 H-\beta I-a^{2}\right)
$$

where $n_{0}$ is the total density, $I$ the kinetic momentum in the z-direction, $\beta$ and $a$ two constant, and $H$ is the transverse hamiltonian

$$
H\left(r, v_{r}, v_{\theta}\right)=\frac{1}{2}\left(v_{r}^{2}+v_{\theta}^{2}\right)+\phi_{s}(t, r)
$$

is a stationary solution of the Vlasov-Poisson equation. The $\mathrm{K}-\mathrm{V}$ distribution is not the best model to represent a laboratory beam but it was studied by many authors and it is the departure point for the focusing ([18]). In fact, to analyze and compare the behavior of different stationary or non stationary distributions, Lapostolle and Sacherer in 1971 introduce RMS quantities and the concept of equivalent beams. According to this concept, two beams composed of the same particles species and having the same current and kinetic energy are equivalent if the second moments of the distribution function are the same. If we consider a normalized stationary or non stationary distribution $f\left(x, y, x^{\prime}, y^{\prime}\right)$ in four-dimensional transverse trace space where $x^{\prime}=v_{x} / v_{z}$ and $y^{\prime}=v_{y} / v_{z}$. The second moment in the particle coordinates $x$ is defined by

$$
\overline{x^{2}}=\int_{\mathbf{R}^{4}} x^{2} f\left(x, y, x^{\prime}, y^{\prime}\right) d x d y d x^{\prime} d y^{\prime},
$$

and the RMS beam width in the $x$-direction is given by

$$
x_{r m s}=\sqrt{\overline{x^{2}}} .
$$

In the same way we can define $y_{r m s}, x_{r m s}^{\prime}$ and $y_{r m s}^{\prime}$. As an example for a $\mathrm{K}-\mathrm{V}$ distribution whose boundary in the $x-x^{\prime}$ plane is described by a ellipse, if we consider a position where the ellipse is upright and let $x_{\max }=a, x_{\max }^{\prime}=a^{\prime}$ denote the maximum $x$-position (radius or envelope) and maximum slope in the particle distribution, then we can show that

$$
\overline{x^{2}}=a^{2} / 4, \quad \overline{x^{\prime 2}}=a^{2} / 4,
$$


Then we introduce the RMS emittance $\epsilon_{x}$ which is a fundamental quantity in beams physics

$$
\epsilon_{x}=\sqrt{\left(\overline{x^{2}} \overline{x^{\prime 2}}-{\overline{x x^{\prime}}}^{2}\right)}
$$

Generally the emittance is not constant and increases and oscillates because of the non linear effects caused by the coupling between the Vlasov equation and the Poisson equation. To simulate the propagation of laboratory beams we use the model of semi-Gaussian beam and Maxwell-Boltzmann beam. In Order to focus these beams we use the the K-V distribution and the concept of equivalent beams. Indeed the parameters of the studied beam is computed so that its moments are the same as those of a $\mathrm{K}-\mathrm{V}$ distribution.

\subsubsection{The discretization of the axisymetric Vlasov equation.}

For a fixed $I$ the characteristic curves are defined by the system of ordinary differential equations

$$
\left\{\begin{array}{l}
\frac{d r}{d t}=v_{r} \\
\frac{d v_{r}}{d t}=F(t, r)=E_{s}(t, r)+\frac{I^{2}}{r^{3}}-\omega_{0}^{2} r .
\end{array}\right.
$$

In order to solve the axisymmetric Vlasov equation we apply our numerical method where we split the advection in physical space from the advection in velocity space. The phase space to discretize is $\left(r, v_{r}, I\right)$. For the subspace $\left(r, v_{r}\right)$ we use an unstructured mesh whose the boundary is a half ellipse. For the discretization of $I$ we use a grid. However we have to choose a judicious discretization in the $I$-direction. Indeed when the total electric field is linear as it is the case for a $\mathrm{K}-\mathrm{V}$ distribution, the characteristic curves can be recast as

$$
\frac{\omega^{2}}{2} r^{2}+v_{r}^{2}+\frac{I^{2}}{r^{2}}=c
$$

where $c$ is a constant and

$$
\frac{\omega^{2}}{2} r^{2}=\left(\frac{\omega_{0}^{2}}{2}-\frac{n_{0}}{4}\right) r^{2}=-\left(\phi_{s}+\phi_{a}\right) .
$$

Then, we must control the ratio $I / r$, and the discretization in the $I$-direction must verify

$$
I= \pm \omega r^{2} .
$$

During the half time step advection in the physical space we see that we need to put an artificial boundary condition for $r=0$. Indeed for $r=0$ and $v_{r}>0$ the particles flux is inward, whereas for $v_{r}<0$ the particles flux is outward, and the particles leave the domain of computation. Then to modelize the 
crossing of particles through the axis $r=0$ we impose a specular reflection as boundary condition for $r=0$

$$
f\left(0, v_{r}, I\right)=f\left(0,-v_{r}, I\right), \quad \forall v_{r}>0 .
$$

During advection in velocity space step we need to compute $\partial_{r} F(t, r)$. Thanks to the Poisson equation (17) we obtain

$$
\frac{\partial F}{\partial r}(t, r)=\rho(t, r)-\frac{E(t, r)}{r}-3\left(\frac{I}{r^{2}}\right)^{2}-\omega_{0}^{2} .
$$

\subsubsection{Semi-Gaussian beam}

The initial data in Cartesian coordinate are

$$
f_{0}\left(x, y, v_{x}, v_{y}\right)=\frac{n_{0}}{\left(2 \pi v_{t h}^{2}\right)\left(\pi a^{2}\right)} \exp \left(-\frac{1}{2 v_{t h}^{2}}\left(v_{x}^{2}+v_{y}^{2}\right)\right)
$$

if $x^{2}+y^{2} \leq a^{2}$ and $f_{0}\left(x, y, v_{x}, v_{y}\right)=0$ if $x^{2}+y^{2}>a^{2}$. The density $n_{0}$ and the pulsation $\omega_{0}$ are compute thanks to RMS quantities so that the semi-Gaussian beam is equivalent to the matched $\mathrm{K}-\mathrm{V}$ beam. In fact we fix the beam radius $a=1$, the thermal velocity $v_{t h}=1$ and the tune depression $\eta=\omega / \omega_{0}=1 / 4$. Then we deduce $\omega_{0}$ and $n_{0}$ by the following formulae

$$
v_{t h}=\frac{1}{2} a \eta \omega_{0}, \quad \omega_{0}=\sqrt{\frac{n_{0}}{2\left(1-\eta^{2}\right)}} .
$$

We observe the formation of a space charged wave which starts at the edge of the beam and propagates inside the beams to be reflected close to the axis $r=0$ (see figures 19. At the beginning the total electric field is linear inside the beam. The variations of the total electric field are small but sufficient to perturb strongly the density (see figures 19 and 20).

\subsubsection{Maxwell-Boltzmann beam}

The initial data are the normalized Maxwell-Boltzmann distribution (which is a stationary solution for the Vlasov-Poisson system)

$$
f_{0}\left(r, v_{r}, v_{\theta}\right)=\frac{\alpha}{2 \pi} \exp \left(-\left(\frac{1}{2}\left(v_{r}^{2}+v_{\theta}^{2}\right)+\phi_{s}(t, r)+\phi_{a}(t, r)\right)\right)
$$

with $\phi_{a}(t, r)=r^{2} / 4, E_{a}(t, r)=-\partial_{r} E_{a}(t, r), 0 \leq \alpha \leq 1$ and where the potential $\phi_{s}$ satisfies the Poisson equation

$$
-\frac{1}{r} \frac{\partial}{\partial r}\left(r \frac{\partial \phi_{s}}{\partial r}\right)=\alpha \exp \left(-\phi_{s}-r^{2} / 4\right)
$$



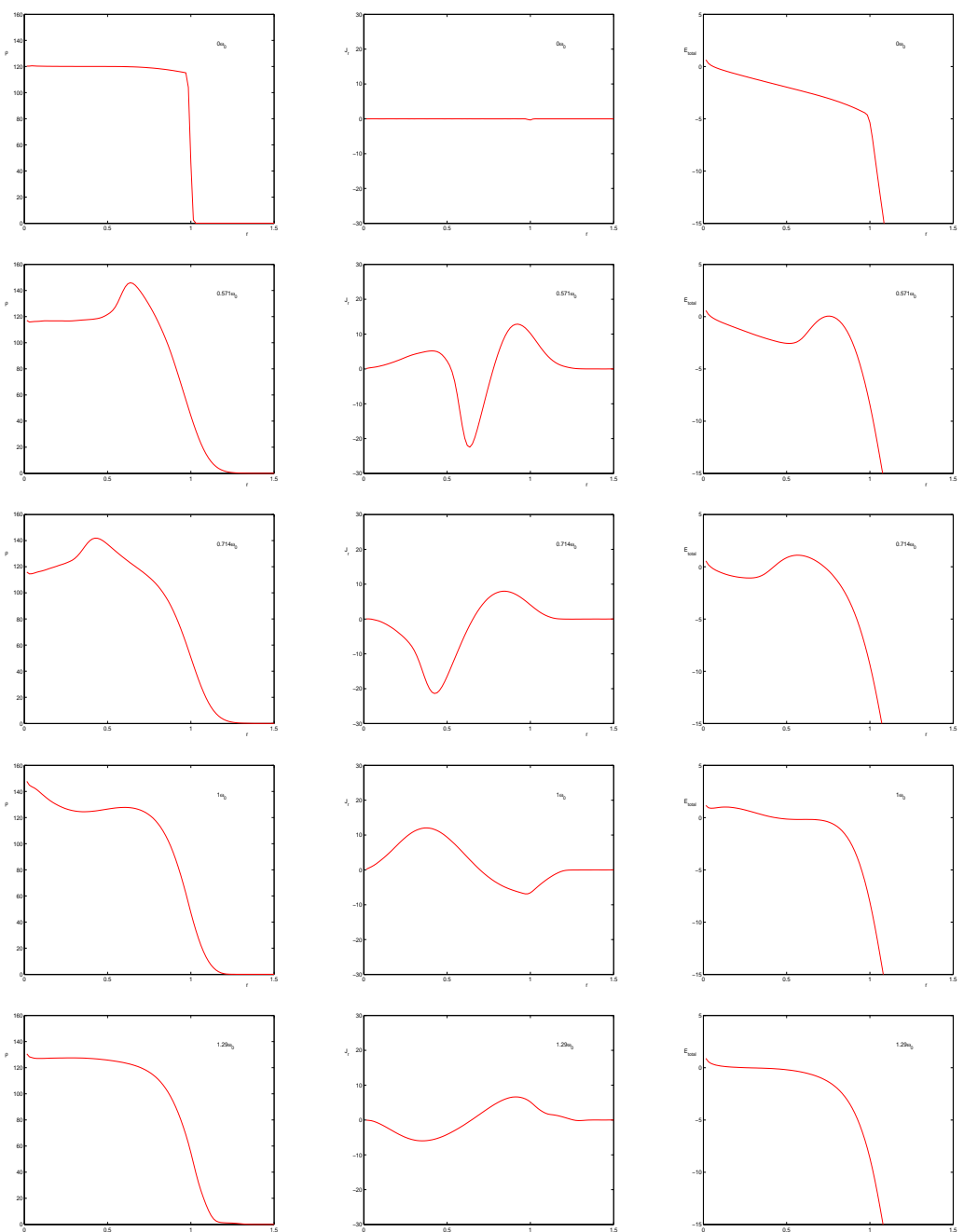

(a) density $\rho(t, r)$

(b) current $J_{r}$

(c) Total field $E_{\text {total }}$

Fig. 19. Evolution of the density $\rho(t, r)$ (a), the current $j_{r}$ (b), and the total force field $E_{\text {total }}=E_{s}(t, r)-\omega_{0}^{2} r$ (c), with the NC1 interpolation operator at times $t=0 \omega_{0}^{-1}, 0.571 \omega_{0}^{-1}, 0.714 \omega_{0}^{-1}, 1 \omega_{0}^{-1}, 1.29 \omega_{0}^{-1}, N_{m e s h}=2509056$ in the case of the semi-gaussian beam

solved numerically by finite differences. Then we perturb the density of $50 \%$. We take $a=1, v_{t h}=1, \eta=1 / 2$ and we match the beam in a similar fashion as semi-Gaussian beam. Figure 22 shows the appearance of a halo around the beam core. Nevertheless we must be careful when we interpret these results because the physical effects we simulate is so fine that numerical effects can distort the solution if we do not take a mesh which is not enough refined. Each figure of the density 22 (b) are obtained when the $v_{r_{r m s}}$ value is an extremum, because this is the time when the halo is the more perceptible. Between two extremun of the $v_{r_{r m s}}$ value we observe the propagation of a space charged 


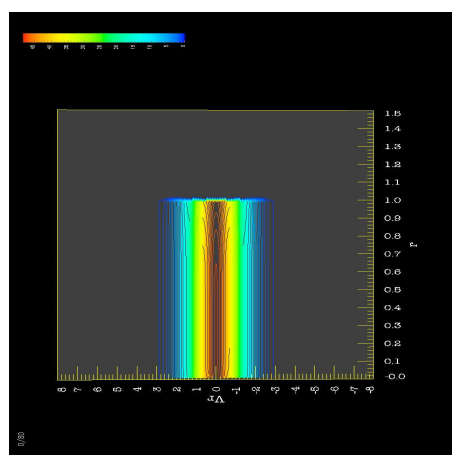

(a) $t=0 \omega_{0}^{-1}$

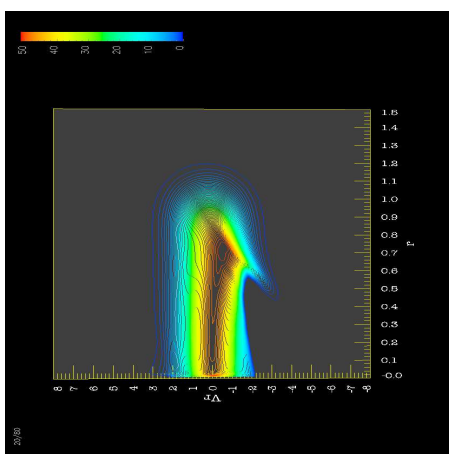

(b) $t=0.571 \omega_{0}^{-1}$

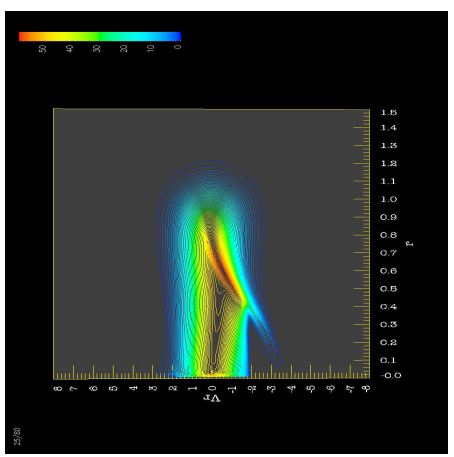

(c) $t=0.714 \omega_{0}^{-1}$

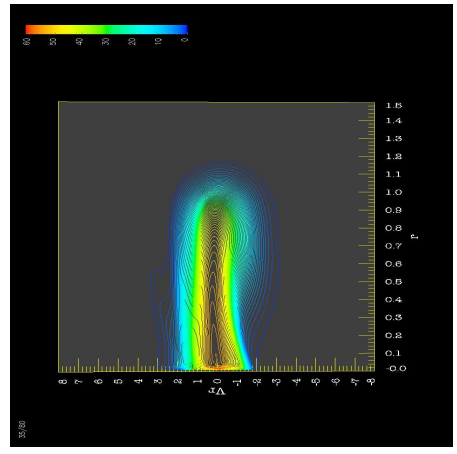

(d) $t=1 \omega_{0}^{-1}$

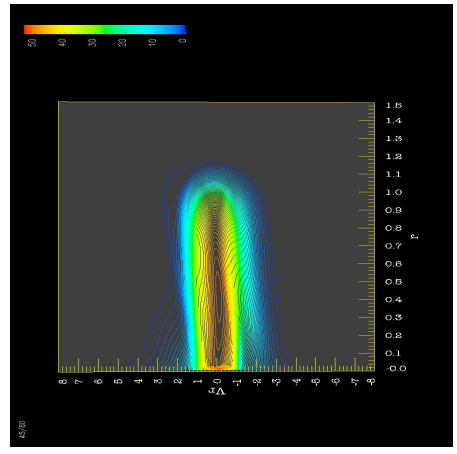

(e) $t=1.29 \omega_{0}^{-1}$

Fig. 20. Evolution of the distribution function $f$ in the phase space $\left(r, v_{r}\right)$ with the NC1 interpolation operator and $N_{\text {mesh }}=2509056$, in the case of the semi-gaussian beam

wave which finishs to be damped down in long time. The density of the halo is about one thousandth of the total density and the halo radius is about 2.5. Even these values seem in agreement with empirical formula of Wangler ([21]) we must take these results with cautiousness. The figures 21 show the evolution of the RMS quantities and the emittance on twenty pulsations. 


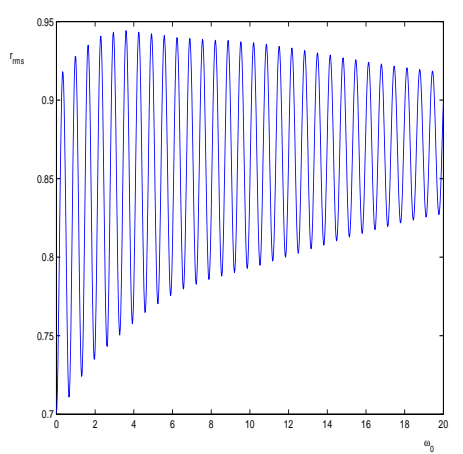

(a) $r_{r m s}$

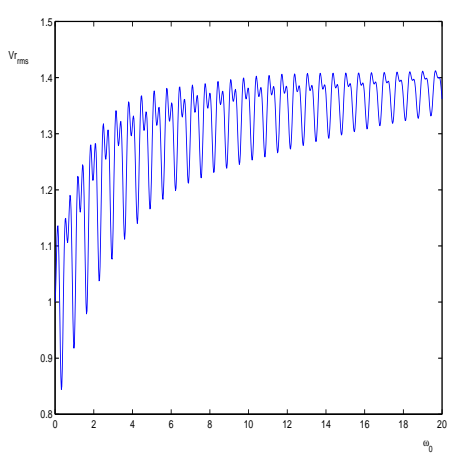

(b) $v_{r_{r m s}}$

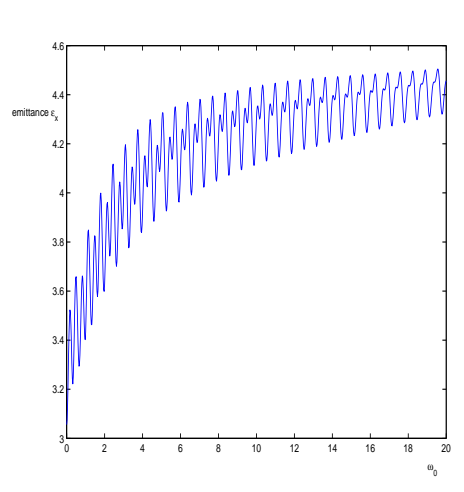

(d) $\epsilon_{x}$

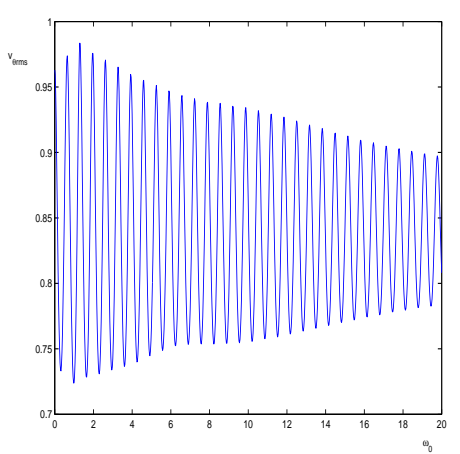

(c) $v_{\theta_{r m s}}$

Fig. 21. Evolution of the RMS radius $r_{r m s}$ (a), $v_{r_{r m s}}$ (b), $v_{\theta_{r m s}}$ (c), and emittance $\epsilon_{x}$ with the NC1 interpolation operator. $T_{\text {final }}=20 \omega_{0}^{-1}, N_{\text {mesh }}=10036224$, in the case of the Maxwell-Boltzmann beam 

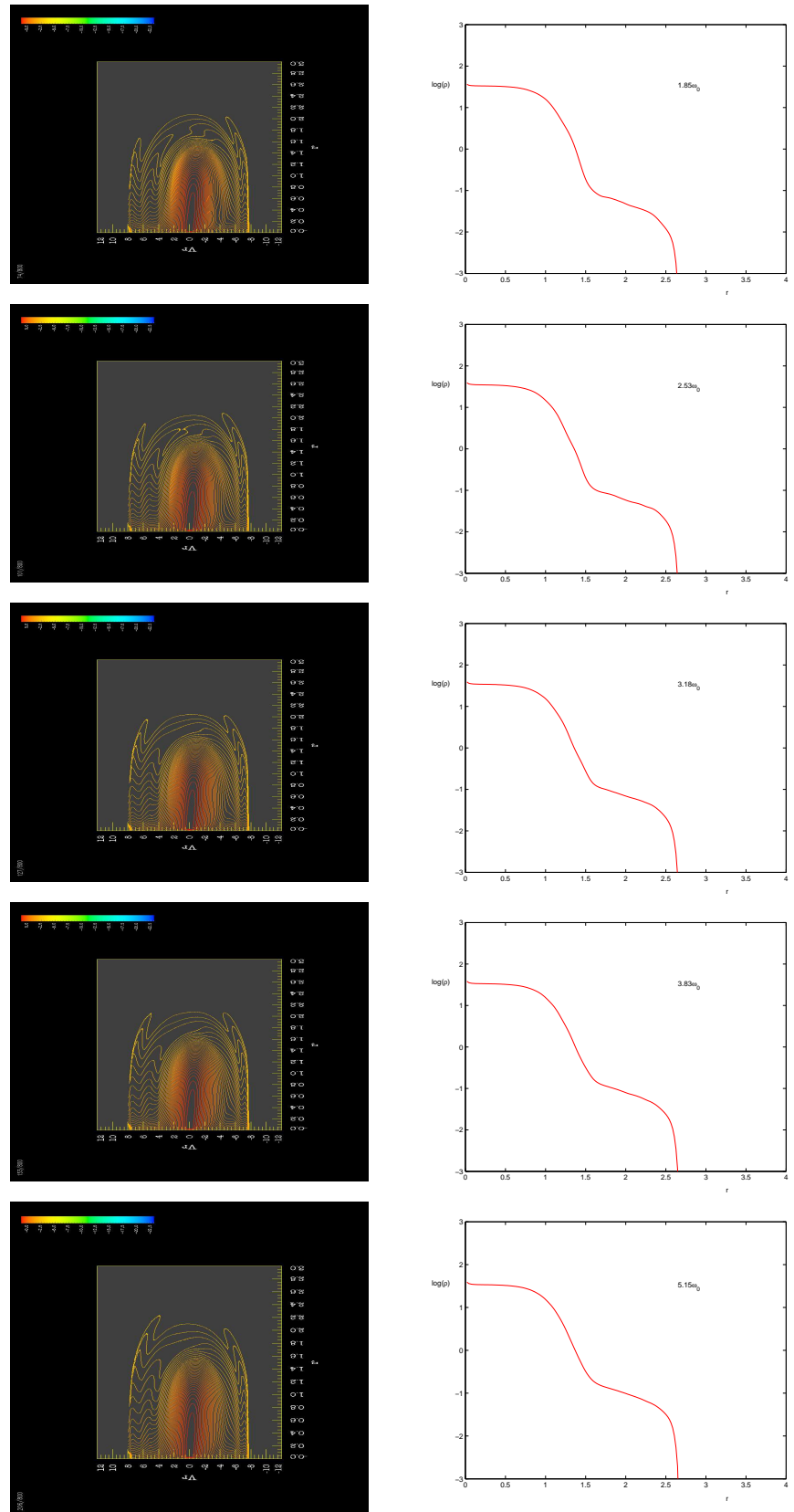

(a) $\log (f)$

(b) $\log (\rho)$

Fig. 22. Evolution of the logarithm of the distribution function $f$ in the phase space $\left(r, v_{r}\right)(\mathrm{a})$, and the logarithm of density $\rho(\mathrm{b})$, with the $\mathrm{NC} 1$ interpolation operator at the time $t=1.85 \omega_{0}^{-1}, 2.53 \omega_{0}^{-1}, 3.18 \omega_{0}^{-1}, 3.83 \omega_{0}^{-1}, 5.515 \omega_{0}^{-1}, N_{m e s h}=10036224$, in the case of the Maxwell-Boltzmann beam 


\section{References}

[1] C.K. Birdsall, A.B. Langdon, Plasma physics via computer simulation McGrawHill, (1985).

[2] J.P. Boris, D.L. Book, Solution of continuity equations by the method of fluxcorrected transport J. Comput. Phys., 20, (1976), 397-431.

[3] F. Bouchut, F. Golse, M. Pulvirenti, Kinetic equations and asymptotic theory, Series in Applied Mathematics, P.G. Ciarlet and P.-L. Lions (Eds.) Gauthiervillars (2000).

[4] F.F. Chen, Introduction to plasma physics, Plenum Press, 1974, New York.

[5] Cheng, G. Knorr, The integration of the Vlasov equation in configuration space, J. Comput. Phys., 22, (1976), 330-351

[6] Handbook of Numerical analysis, Finite element methods (part 1),Vol II, P.G. Ciarlet and J.L. Lions (Eds.) North-Holland (1991).

[7] J.L Delcroix, A. Bers, Physique des plasmas, Tome 1 \& 2 Savoirs Actuels, CNRS édition, (1994).

[8] E. Fijalkow, A numerical solution to the Vlasov equation, Comput. Phys. Commun., 116, (1999), 319-328.

[9] F. Filbet, E. Sonnendrücker, P. Bertrand Conservative numerical schemes for the Vlasov equation, J. Comput. Phys., 172, (2001), 166-187.

[10] R.W. Hockney, J.W. Eastwood, Computer simulation using particles, McGrawHill, 1981.

[11] A. J. Klimas, W. M. Farell, A splitting algorithm for Vlasov simulation with filamentation filtration, J. Comp. Phys., 110 (1994), 150.

[12] R. J. Leveque, Numerical methods for conservation laws, Lectures in Mathematics : ETH Zürich, Birkhäuser, 1992.

[13] E. Lifchitz, L. Pitayevski, Cinétique physique, Tome 10, ed. MIR 1990.

[14] G. Manfredi, Long time behaviour of non linear Landau damping, Phys. Rev. Letters, 79, (1997), 2815-2818.

[15] P. Mineau, Simulation en physique des plasmas, PHD thesis, Orléans, France (1997).

[16] T. Nakamura, T. Yabe, The cubic interpolated propagation scheme for solving the hyper-dimensional Vlasov-Poisson equation in phase space, Compt. Phys. Comm., 120, (1999), 122-154.

[17] A. Priestley, A quasi-conservative version of the semi-lagrangian advection scheme, Mont. Weath. Rev., (1992) 621-629. 
[18] M. Reiser, Theory and design of charged particle beams Wiley series in beam physics and accelerator technology, (1994).

[19] S. Gravel, A. Staniforth, A Mass-conserving Semi-Lagrangian scheme for the Shallow-Water Equations, Mont. Weath. Rev., (1994) 243-248.

[20] E. Sonnendrücker, J. Roche, P. Bertrand, A. Ghizzo, The semi-Lagrangian method for the numerical resolution of Vlasov equations, J. Comput. Phys., 149 (1996), 841-872.

[21] T.P. Wangler, R.W. Garnett, E.R. Gray, R.D. Ryne, T.S. Wang, Dynamics of beam halo in mismatched beams, XVIII International Linac Conference, Geneva, Switzerland, (1996).

[22] S. I. Zaki, L. R. Gardner, T. J. M. Boyd A finite element code for the simulatiom of one-dimensional Vlasov plasmas I Theory, J. Comput. Phys., 79 (1988), 184, II Applications, J. Comput. Phys., 79 (1988), 200. 